\title{
Finite element modeling of multi-pass welding and shaped metal deposition processes
}

\author{
Michele Chiumenti a,*, Miguel Cervera ${ }^{\mathrm{a}}$, Alessandro Salmi ${ }^{\mathrm{b}}$, Carlos Agelet de Saracibar ${ }^{\mathrm{a}}$, \\ Narges Dialami ${ }^{\text {a }}$, Kazumi Matsui ${ }^{\mathrm{c}}$
}

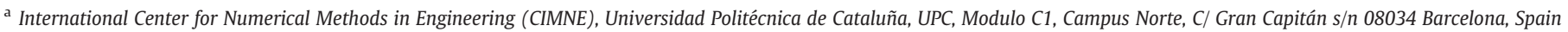

b Politecnico di Torino, Department of Production Systems and Business Economics (DISPEA), Corso Duca degli Abruzzi 24 - 10129 Torino, Italy

c Yokohama National University, Graduate School of Environment and Information Science, Tokiwadai 79-7, Yokohama, Japan

\section{A R T I C L E I N F O}

\section{Article history:}

Received 17 July 2009

Received in revised form 25 February 2010

Accepted 26 February 2010

Available online 14 April 2010

\section{Keywords:}

Shaped metal deposition process

Multi-pass welding

Hot-cracking

Thermo-mechanical analysis

Finite element method

\begin{abstract}
A B S T R A C T
This paper describes the formulation adopted for the numerical simulation of the shaped metal deposition process (SMD) and the experimental work carried out at ITP Industry to calibrate and validate the proposed model. The SMD process is a novel manufacturing technology, similar to the multi-pass welding used for building features such as lugs and flanges on fabricated components (see Fig. $1 \mathrm{a}$ and $\mathrm{b}$ ). A fully coupled thermomechanical solution is adopted including phase-change phenomena defined in terms of both latent heat release and shrinkage effects. Temperature evolution as well as residual stresses and distortions, due to the successive welding layers deposited, are accurately simulated coupling the heat transfer and the mechanical analysis. The material behavior is characterized by a thermo-elasto-viscoplastic constitutive model coupled with a metallurgical model. Nickel super-alloy 718 is the target material of this work. Both heat convection and heat radiation models are introduced to dissipate heat through the boundaries of the component. An in-house coupled FE software is used to deal with the numerical simulation and an ad-hoc activation methodology is formulated to simulate the deposition of the different layers of filler material. Difficulties and simplifying hypotheses are discussed. Thermo-mechanical results are presented in terms of both temperature evolution and distortions, and compared with the experimental data obtained at the SMD laboratory of ITP.
\end{abstract}

(c) 2010 Elsevier B.V. All rights reserved.

\section{Introduction}

Automotive, aeronautical and naval industries use different kinds of welding technologies when assembling or joining structural components.

As an example, aero-gas turbine casings are designed to contain the gas stream through the compressor and turbine sections of the engine core. This involves the fabrication of anular structures offering the minimum of clearance between the internal casing wall and the rotating turbo machinery in order to optimize aerodynamic flow and thereby the ultimate thrust and the fuel efficiency. Complex structures such as flanges and bosses are necessary for joining casing sections or supporting peripherical components, and due to their complexity they have traditionally been manufactured by casting or forging techniques. However, those structures must also provide good mechanical behavior to ensure structural integrity to prevent mechanical failure. As an alternative to the casting technology, the shaped metal deposition process (SMD) has been proposed to enhance the mechanical properties of the material due to the different solidification conditions inherent to the deposition process. The SMD is a manufacturing technology,

\footnotetext{
* Corresponding author.

URL's:URL: michele@cimne.upc.edu, http://www.cimne.com (M. Chiumenti).
}

originally patented by Rolls-Royce plc, similar to multi-pass welding, which can be used to build components such as flanges or lugs directly on fabricated components. Parts are built layer-by-layer depositing the melted material along a predefined welding path. At each pass a new layer is deposited on the previous substrate allowing the fabrication of the desired geometry (see Fig. 1a-b).

This innovative technique has been considered of great interest due to the possibility of employing standard welding equipment without the need for extensive new investment [30,31]. Typically, it is considered that MIG welding may be attractive for the SMD process because it offers high power densities at low cost. As an alternative, TIG welding machines linked to wire feeding systems show advantages for additive manufacturing processes allowing more flexible feeding angles. Nowadays, SMD technique is evolving towards mixed laser and electron beam welding-machines which allow a better control of the heat flux.

During the SMD process the solidification occurs rapidly in a small and localized volume, resulting in a fine as deposited microstructure. The filler material is cooled mainly by conduction through the (much cooler) substrate, leading to very high solidification rates. On one hand, this is an advantage over alternative manufacturing processes such as casting, forging or machining because the quality of the final component presents a much finer grain size as well as the reduction of both the buy- 
a

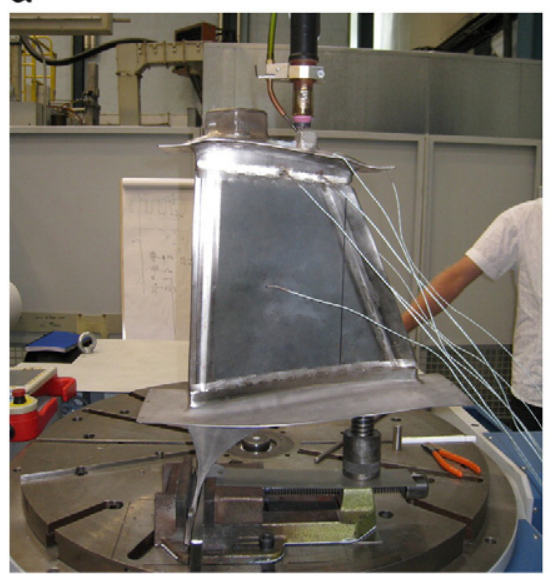

b

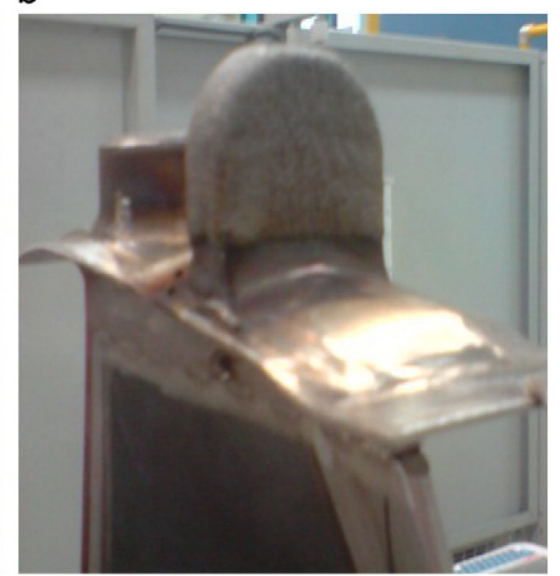

Fig. 1. a) SMD on a real aircraft component, and b) detail of the SMD.

to-fly ratio and the production time. On the other hand, the base material is heated up at each welding pass so that the temperature history of a point within the heat affected zone (HAZ) suffers a cyclic temperature evolution. This temperature evolution may lead to the remelting of the material and, in any case, it induces a continuous change in the microstructure during the full process. Irregular liquation and cracking in the HAZ is an undesired phenomenon which becomes a drawback of the SMD process. The combination of liquid films along

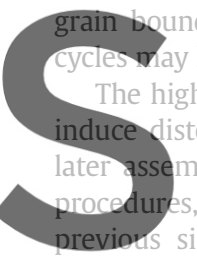
indaries and thermal (tensile lead to the formation of irreg
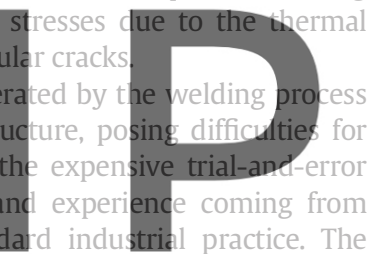

numerical simulation of the process is a very interesting alternative to optimize the welding strategy. Both the thermal and the mechanical

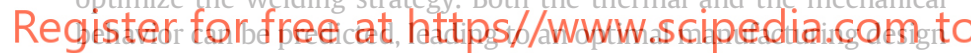
for large welded structures.

The objective of this work is the accurate numerical simulation of the SMD process to be able to analyse both the temperature evolution and the stress field generated during the process, allowing the estimation of the hot-cracking risk as well as getting a useful tool for the manufacturing design optimization.

To this end, the paper presents a detailed description of the FE technology used to simulate the deposition of the material during the welding process. The strategy adopted consists on an element activation procedure, which allows switching on the elements according to the welding path defined by the user together with a constitutive model able to describe the material behavior in all the temperature range of the process.

The outline of the paper is as follows. Firstly, the heat transfer model is introduced including the phase transformation phenomena and the heat source due to the welding arc. Secondly, a mechanical constitutive model suitable for all the different phases of the material, from the initial liquid phase to the final solid state passing through the so called mushy zone where the phase transformation takes place is presented. The mechanical model is enhanced by a continuous damage model able to represents the porosity induced by the hotcracking phenomena. Later, the variational formulation is discussed stressing out the necessity of an element technology to deal with the incompressible behavior of the material. The element activation technology required to simulate the material deposition is discussed in detail. Finally, different numerical simulations of both welding and SMD processes are presented to assess the present formulation compared to the experimental measurements carried out at ITP.

\section{Heat transfer analysis}

The local governing equation for the thermal problem is the balance of energy equation. This equation controls the temperature and the solidification evolution and it can be stated as a function of the enthalpy state variable per unit of volume $H$ as:

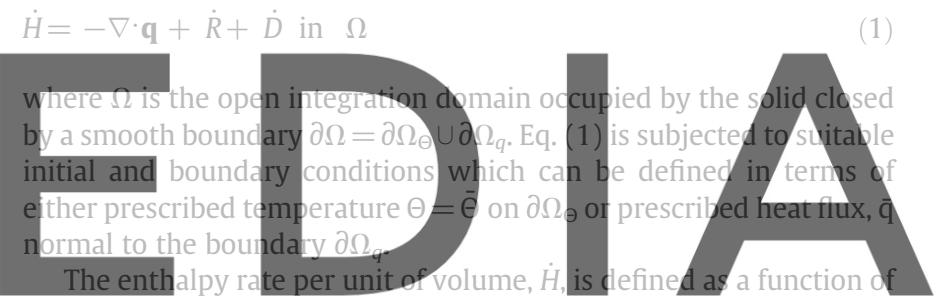

the temperature field, $\Theta$, as:

\section{diownload the version without the watermark}

where $C_{\Theta}(\Theta)$ is the (temperature dependent) heat capacity coefficient and $\mathrm{L}_{p c}(\Theta)$ is the rate of latent heat released during the solidification process. The heat flux per unit of surface $\mathbf{q}$ is computed as a function of the temperature gradient through the Fourier's law as:

$\mathbf{q}=-k_{\Theta} \nabla \Theta$

where $k_{\Theta}(\Theta)$ is the (temperature dependent) heat conductivity.

On one hand, when studying the SMD process, the thermomechanical dissipation rate per unit of volume $\dot{D}$ can be neglected in front of the heat flux generated by the thermal gradient between the melted material and the component. On the other hand, even if the heat conduction drives the solidification and cooling evolution due to the high conductivity of the metallic materials, the radiation and the convection heat fluxes at the boundary interface, $q_{\text {rad }}$ and $q_{\text {conv }}$, respectively, cannot be neglected [8].

The heat radiation flux, $q_{\text {rad }}$, is possibly the most important boundary condition of the problem, especially at the highest temperature of the filler material during the metal deposition. The radiation heat flux can be computed using the Stefan-Boltzmann law as a function of the surface temperature, $\Theta$, and the room temperature, $\Theta_{e n v}$ as:

$q_{\text {rad }}=\sigma_{\text {rad }} \varepsilon_{\text {rad }}\left(\Theta^{4}-\Theta_{\text {env }}^{4}\right)$

where $\sigma_{\text {rad }}$ is the Stefan-Boltzmann constant and $\varepsilon_{\text {rad }}$ is the emissivity correction factor, respectively. It must be pointed out that the radiation heat flux can be neglected out of the HAZ. This means that 
its global effect can be accounted for by modifying the efficiency parameter of the heat source without loss of accuracy in the simulation.

The heat dissipated by convection, $q_{\text {conv }}$, can be estimated using the Newton's law, as:

$q_{\text {conv }}=h_{\text {conv }}\left(\Theta-\Theta_{\text {env }}\right)$

where $h_{\text {conv }}(\Theta)$ is the convection heat transfer coefficient.

\section{The phase change transformation}

The solidification of the feeding material, once deposited, is described by a liquid-to-solid phase change transformation. The rate of latent heat released during this solidification process can be described using the following latent heat function:

$\dot{L}_{p c}(\Theta)=L_{p c} \dot{f}_{L}(\Theta)$

where $L_{p c}$ is the total amount of latent heat released/absorbed during the phase-change, $f_{L}(\Theta)=1-f_{S}(\Theta)$ is the liquid fraction function and $f_{S}(\Theta)$ is the solid fraction.

The reference material in this work is the nickel super-alloy 718. This material is one of the nickel-base alloys frequently used in the aerospace industry, particularly for turbine components, having a combination of high strength at moderate temperatures and a good creep and fatigue resistance. Furthermore the material is outstanding regarding oxidation and corrosion resistance features making it well suited for service in extreme envitonments. In practice, nickel-base alloys have an excellent
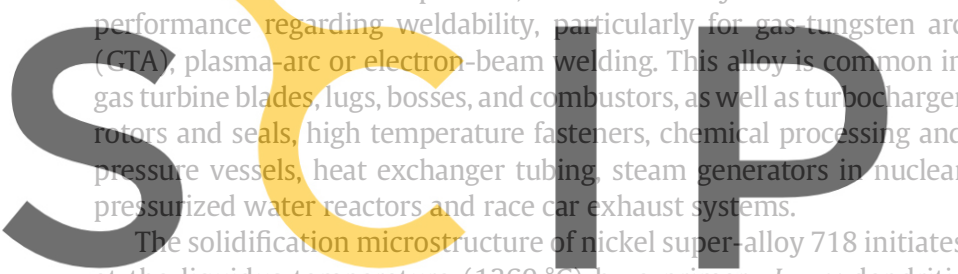

at the liquidus temperature $\left(1360^{\circ} \mathrm{C}\right.$ ) by a primary $L \rightarrow \gamma$ dendritic reaction, in which the interdendritic liquid is enriched in $\mathrm{Nb}$ and $\mathrm{C}$.

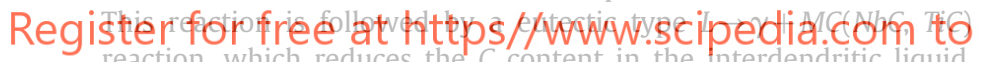
reaction, which reduces the $C$ content in the interdendritic liquid. These types of carbides frequently solidify intergranularly during long aging times and stress conditions [13]. Finally, at lower values of C/Nb ratios, a second eutectic type $L \rightarrow \gamma+$ Laves occurs reducing the $N b$ content. At very high cooling rates, the small amount of $\gamma+M C$ carbides can be ignored so that the solidification can be represented as a binary eutectic $\gamma /$ Laves phases [14] as presented in Fig. 2.

Denoting $C_{0}$ as the nominal alloy composition $(\mathrm{Nb})$ and $C_{L}, C_{S}$ as the instantaneous liquidus and solidus compositions, respectively, then it is possible to write:

$f_{S} C_{S}+f_{L} C_{L}=C_{0}$

and differentiating:

$\left(C_{L}-C_{S}\right) d f_{S}=f_{S} d C_{S}+f_{L} d C_{L}$

It is reasonable to assume no solid state diffusion $\left(f_{S} \mathrm{~d} C_{S}=0\right)$ during the solidification, especially at the very high cooling rates induced by the welding (SMD) process, no dendritic branching, and that the thermodynamic equilibrium is maintained at the moving solid/liquid interface. This given, the alloying element concentration profile between the cell core and the interdendritic region can be described by the Scheil's rule by integrating the above equation:

$C_{L}=C_{0}\left(1-f_{S}\right)^{(k-1)}$

where $k=C_{S} / C_{L}$ is the partition coefficient, that is, the ratio between the composition of the solid and the composition of the liquid at

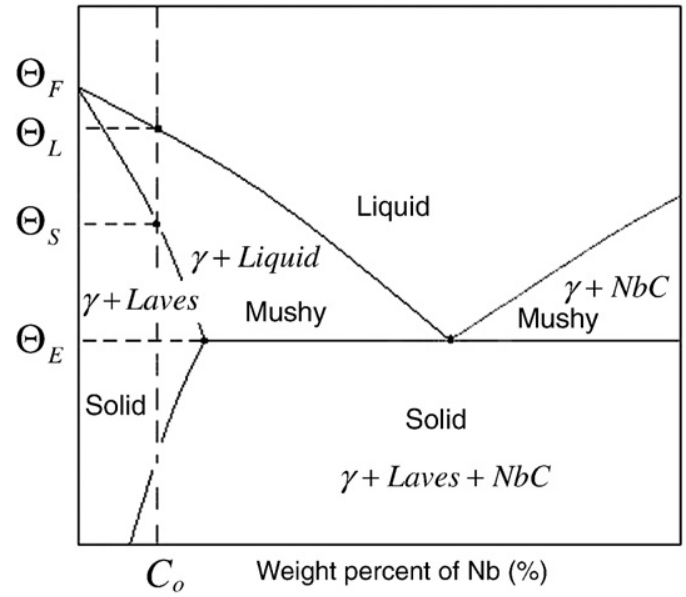

Fig. 2. Phase diagram for Nickel alloy-718.

equilibrium. As a further hypothesis, it is possible to consider $k=$ const, that is not significantly different from the beginning until the end of the solidification, leading to:

$$
C_{L}(\Theta)=C_{0}\left[\frac{\Theta_{F}-\Theta}{\Theta_{F}-\Theta_{L}}\right] ; \quad C_{S}(\Theta)=C_{0}\left[\frac{\Theta_{F}-\Theta}{\Theta_{F}-\Theta_{S}}\right]
$$

where $\Theta_{F}$ is the fusion temperature.

As a result, it is possible to study the solidification process (Scheil's

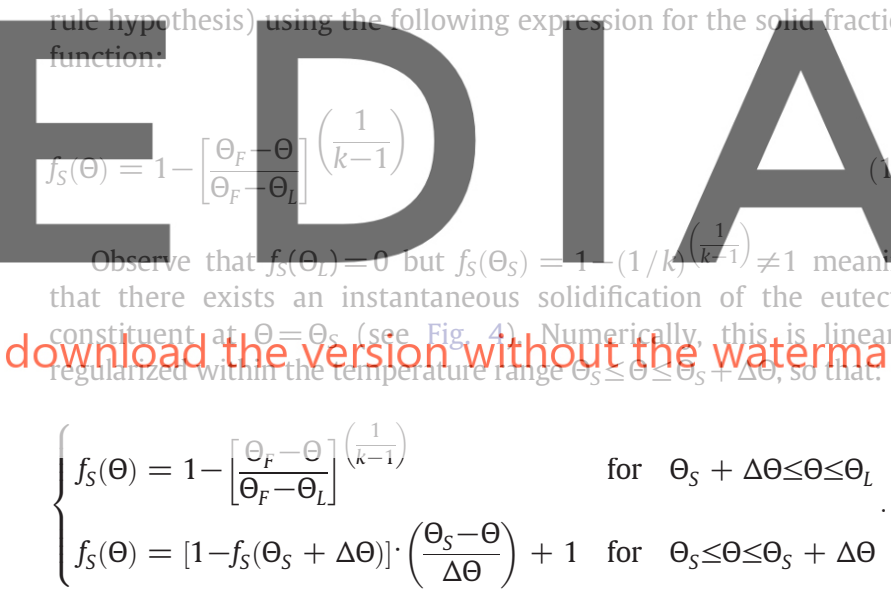

\section{The heat source}

The source term, $\dot{R}$, which appears in Eq. (1), is one of the key points when studying the SMD process. In the literature it is possible to find different models to represent the welding heat source with different degrees of sophistication.

A first level of complexity, the mathematical model proposed by Rosenthal [9] considers a moving heat flux on a 3D geometry. It is possible to choose a more accurate characterization of the energy input defining a Gaussian power distribution for a circular welding pool as presented by Pavelec et al. in [10]. The double ellipsoidal power density distribution presented in [11] by Goldak et al. is the most sophisticated model currently proposed.

There exist technical difficulties from the numerical point of view to adopt such a detailed power distribution. The mesh density generally used to discretize both the SMD material and the metal sheet below, is not fine enough to define a complex welding pool shape or a nonuniform heat source. This is only done if the simulation of the welding pool is the objective itself. If the global structure must be considered, the 


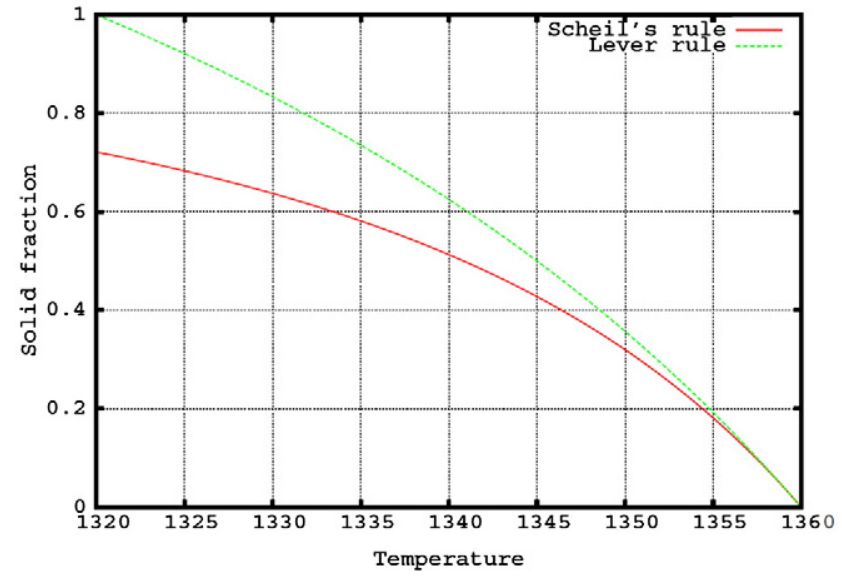

Fig. 3. Scheil's rule and lever rule to describe the solidification evolution.

size of heat source is of the same dimension than the element size generally used for a thermo-mechanical analysis. Therefore, when the global model must be taken into account, the resulting mesh density is usually too coarse to represent the actual shape of the heat source [12].

It is important to remark that when the filler material is used in the welding process, a large percentage of the energy dispensed by the welding arc is used to melt that material and it is only partially transmitted to the base. Experimentally, it can be also observed that after melting, the filler material forms a welding pool, which covers the base material. Due to the over-heating base material is also partially melted an generated in the welding pool mixes feeding system is heated-up to the melt rial; later, the welding pool is t
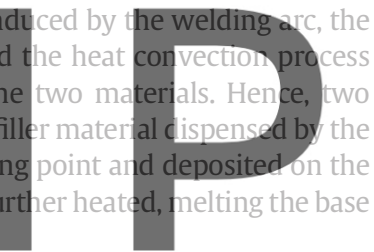

It can be noted that the numerical model could set the temperature at the welding pool as the initial temperature of the deposited material.

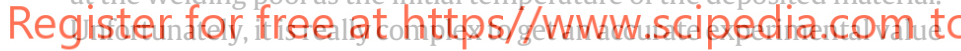
for this temperature because any temperature measurement is distorted by the heat flux of the gas close to the welding arc: approximately $10.00{ }^{\circ} \mathrm{C}$. The only accurate input data is the power of the welding arc, which controls the amount of energy introduced into the system.

The welding arc power is defined by the arc voltage, $V$ and the welding current, $I$, as:

$\dot{P}=\eta \cdot V I$

where $\eta$ is the welding efficiency.

Hence, when a filler material is used in the welding process, the definition of a uniform volume heat source seems to be a convenient solution to heat up the material to be deposited along the welding path. Note that this heat source is applied just to the volume deposited within the current time step (filler material) and is moving according to the metal deposition procedure. Therefore, the simulation strategy, based on finite element discretization, must combine the searching technique used to find out the elements below the welding arc together with the definition of an impulse thermal loading (the applied heat source) at each time-increment of the simulation.

The power density, $\dot{R}$, is computed as:

$\dot{R}=\frac{\dot{P}}{V_{M D}}$

where $V_{M D}$ is the volume of the SMD layer deposited during the current time-step. In a numerical simulation, this volume depends on the FE discretization, leading to an approximation error which increases when coarse meshes are adopted. As an alternative, the volume of the filler material melted by the feeding system during the same time-step, $V_{\text {feed }}$, is a more convenient choice to be used as a reference volume when computing the power density:

$\dot{R}=\frac{\dot{P}}{V_{\text {feed }}}=\frac{\eta \cdot V I}{V_{\text {feed }}}$

A final feature, to be taken into account, is the thermal expansion that the material experiences when its temperature increases from the room temperature to the melting temperature. During the process, this expansion is experimented by the wire when it passes through the welding arc. Later on, the material is deposited and its temperature decreases provoking a thermal contraction of the material. In the numerical model the activated elements are not free to expand during the heating phase because their nodes are shared with the layers of the material below it. To avoid the generation of spurious thermal stresses, the initial temperature of the elements to be activated is set to the melting (liquid) temperature, $\theta_{L}$, so that any temperature increment above this value does not induce any thermal deformation (see full details in the next section). Consequently, Eq. (15) should be corrected to remove the energy rate which corresponds to the adiabatic temperature increment from the room to the melting temperature, that is

$\dot{R}_{\text {melt }}=C_{\Theta}\left(\Theta_{L}-\Theta_{\text {ref }}\right) / \Delta t$.

For the actual values of the material parameters and the standard setting of the welding arc, yields $\dot{R}_{\text {melt }} \approx 2.5 \% \dot{R}$, which is negligible if
compared to the power density of the welding arc.
5. ivechanicall analysis
The mechanical problem is governed by the balance of momentum
equation. The local form, for the olasi-static problem,can be stated as:
find the displacement field, u, for given (prescribed) body forces $\mathbb{f}$, such that:

\section{download the version without the watermark $\nabla \cdot \sigma+\mathbf{f}=\mathbf{0}$ in $\Omega$

where $\nabla \cdot(\cdot)$ is the divergence operator and $\boldsymbol{\sigma}=\boldsymbol{\sigma}(\mathbf{u})$ denotes the Cauchy stress tensor. Additionally, suitable Dirichlet and Neumann conditions must be supplied on the boundary $\partial \Omega=\partial \Omega_{u} \cup \partial \Omega_{t}$. In the following, we will assume these in the form of prescribed displacements $\mathbf{u}=\overline{\mathbf{u}}$ on $\partial \Omega_{u}$ and prescribed tractions $\overline{\mathrm{t}}$ on $\partial \Omega_{t}$.

The stress tensor $\boldsymbol{\sigma}$ is split into its hydrostatic (pressure) and deviatoric parts, $p=\frac{1}{3} \operatorname{tr}(\boldsymbol{\sigma})$ and $\mathbf{s}=\operatorname{dev}(\boldsymbol{\sigma})$, respectively, as $\boldsymbol{\sigma}=p \cdot \mathbf{I}+$ s. This is a very convenient choice to deal with the incompressibility behavior, which is a characteristic of the liquid phase or, more generally, when the deformations are mainly deviatoric as for the classical elasto-visco-plastic model defined for a metallic alloy in the following section.

This given, the local form of the continuum mechanical problem can be stated as: find the displacement field, $\mathbf{u}$, and the pressure field, $p$ (defined as an independent variable of the problem), such that:

$\left\{\begin{array}{l}\nabla \cdot \mathbf{s}(\mathbf{u})+\nabla p+\mathbf{f}=\mathbf{0} \\ \nabla \cdot \mathbf{u}-e^{\Theta}-\frac{p}{K}=0\end{array}\right.$

in $\Omega$

where $e^{\Theta}$ is the thermal deformation which is defined in the next section according to the constitutive equation. Note that this mixed $\mathbf{u} / p$ formulation of the mechanical problem is equivalent to Eq. (17) and it is valid for both the compressible and incompressible cases. In particular, when the material is liquid, no thermal deformation is 
allowed $\left(e^{\Theta}=0\right)$ and the bulk modulus (also referred to as compressibility modulus) $K \rightarrow \infty$ so that Eq. (18)-b enforces the volumetric constraint as:

$\nabla \cdot \mathbf{u}=0 \quad$ in $\Omega$.

In the following section it will be detailed the constitutive law for the stress tensor $\boldsymbol{\sigma}$ (in terms of $p$ and $\mathbf{s}$, respectively) suitable in all the temperature range of the process.

\section{Mechanical constitutive laws}

The mechanical model chosen to simulate the constitutive behavior of the material is based on previous developments in the field of coupled thermo-mechanical analysis for casting processes [6-8]. In both casting and welding processes the temperature range varies from room temperature to very high temperatures above the melting point. As a consequence, the material response should vary from an elasto-plastic behavior when the material is at room temperature to a pure viscous behavior above the melting point. This evolution can be simulated with a thermo-elasto-visco-plastic constitutive model, which includes both isotropic hardening and thermal softening. The definition of temperature dependent material properties (the elastic limit among them) allows a gradual contraction of the von Mises yield-surface as the temperature increases. When the temperature is close to the melting point, the viscous behavior becomes more and more predominant, the elastic limit gradually reduces, vanishing when liquidus temperature is reached. As a result, a purely viscous (Norton-Hoff) model is recovered

\section{when liquid-like behavior must be sinfula}

the solid phase to the liquid-like beha

dependency of the material propert phase change transformation accordi
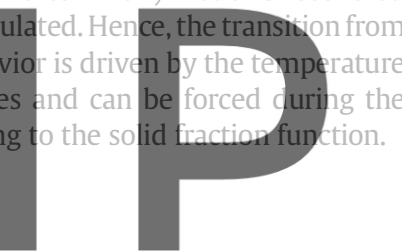

The material behavior in all the temperature range below the solidus temperature is characterized by a thermo-elasto-visco-plastic

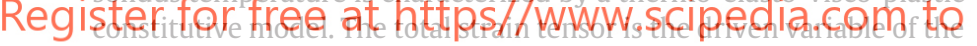
mechanical problem computed in terms of the displacement field as: $\varepsilon=\nabla^{S}(\mathbf{u})$. The constitutive model can be written as:

$\left\{\begin{array}{l}p=K\left(\nabla \cdot \mathbf{u}-\varepsilon^{\Theta}\right) \\ \mathbf{s}=2 G \operatorname{dev}\left(\boldsymbol{\varepsilon}-\boldsymbol{\varepsilon}^{v p}\right)\end{array}\right.$

where $G(\Theta)$ is the (temperature dependent) shear modulus and $K(\Theta)$ is the (temperature dependent) bulk modulus which controls the material compressibility.

The thermal deformation (a volumetric term), $\varepsilon^{\Theta}$, is computed adding the thermal shrinkage characteristic of the change of phase, $\varepsilon^{p c}$, to the thermal contraction during the cooling process from the solidus to room temperature, $\varepsilon^{\text {cool }}(\Theta)$, as follows:

$\varepsilon^{\Theta}(\Theta)=\varepsilon^{c o o l}(\Theta)+\varepsilon^{p c}$

where $\varepsilon^{p c}$ and $\varepsilon^{\text {cool }}(\Theta)$ are computed as:

$$
\left\{\begin{array}{l}
\varepsilon^{p c}=\frac{\Delta V^{p c}}{V_{0}}=\frac{\rho_{S}-\rho_{L}}{\rho_{S}} \\
\varepsilon^{\text {cool }}(\Theta)=3\left[\alpha(\Theta)\left(\Theta-\Theta_{\text {ref }}\right)-\alpha\left(\Theta_{0}\right)\left(\Theta_{0}-\Theta_{r e f}\right)\right]
\end{array}\right.
$$

where $\alpha(\Theta)$ is the (temperature dependent) thermal expansion coefficient while $\rho_{S}=\rho\left(\Theta_{S}\right)$ and $\rho_{L}=\rho\left(\Theta_{L}\right)$ are the material density at solidus and liquidus temperature, respectively. The equation used to compute $\varepsilon^{p c}$ can be deduced from the principle of mass conservation introducing the volume shrinkage, $\Delta V^{p c}$, induced by the change of density during the solidification.

The constitutive model considers a temperature dependent J2yield-surface $\Phi^{v p}(\mathbf{s}, q, \Theta)$ defined as:

$\Phi^{v p}(\mathbf{s}, q, \Theta)=\|\mathbf{s}\|-R(q, \Theta) \leq 0$

where $R(q, \Theta)$ is the (temperature dependent) yield-surface radius defined as:

$R(q, \Theta)=\sqrt{\frac{2}{3}}\left[\sigma_{0}(\Theta)-q\right]$.

The visco-plastic strain tensor (purely deviatoric term), $\boldsymbol{\varepsilon}^{v p}$, together with the evolution laws for the isotropic strain-hardening variable $\xi$, are derived from the principle of maximum plastic dissipation as [17]:

$$
\begin{aligned}
& \dot{\varepsilon}^{v p}=\gamma^{v p} \frac{\partial \Phi^{v p}(\mathbf{s}, q, \Theta)}{\partial s}=\gamma^{v p} \mathbf{n} \\
& \dot{\xi}=\gamma^{v p} \frac{\partial \Phi^{v p}(s, q, \Theta)}{\partial q}=\gamma^{v p} \sqrt{\frac{2}{3}}
\end{aligned}
$$

where $\mathbf{n}=\frac{\mathbf{s}}{\|\mathbf{s}\|}$ and $\gamma^{v p}=\left\langle\frac{\Phi^{v p}(\mathbf{s}, q, \Theta)}{\eta}\right\rangle$ are the normal to the yield surface and the visco-plastic multiplier, respectively, when a ratedependent evolution law is assumed.

The stress-like variable, q, conjugate to the isotropic strain-
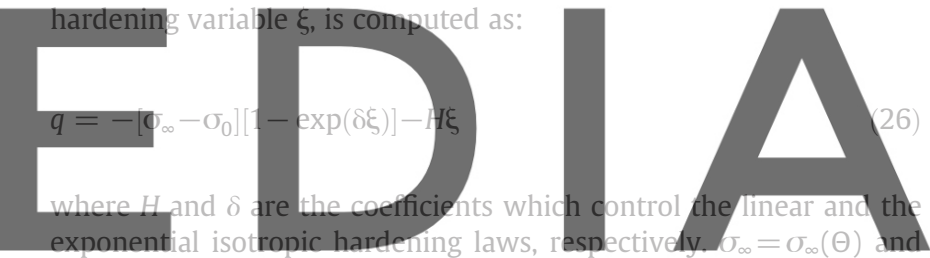

$\sigma_{0}=\sigma_{0}(\Theta)$ are the (temperature dependent) saturation and initial

download the version without the watermark 6.2. Mushy state $\left(\Theta_{S}<\theta<\Theta_{L}\right)$

In this temperature range the material transforms from liquid to solid or vice-versa. The material behavior can be controlled using the solid fraction function: in the limit case, when $f_{S}\left(\Theta_{S}\right)=1$, the model should return to the thermo-elasto-visco-plastic model proposed for the solid phase. On the other hand, when $f_{S}\left(\Theta_{L}\right)=0$ the purely viscous behavior is expected. This transition can be achieved modifying the equations that characterize the solid phase model as follows [6,7]. The constitutive model can be written as:

$$
\left\{\begin{array}{l}
p=\hat{K}\left(\nabla \cdot \mathbf{u}-\varepsilon^{\Theta}\right) \\
\mathbf{s}=2 \hat{G} \operatorname{dev}\left(\boldsymbol{\varepsilon}-\boldsymbol{\varepsilon}^{v p}\right)
\end{array}\right.
$$

where $\hat{K}(\Theta)=\frac{K(\Theta)}{f_{S}(\Theta)}$ is a modified bulk modulus which controls the material compressibility. On one hand, when $f_{S}\left(\Theta_{L}\right)=0$ no thermal deformations are allowed and the incompressible behavior is recovered, as $\nabla \cdot \mathbf{u}=0$. On the other hand, $\hat{G}(\Theta)=\frac{G(\Theta)}{f_{S}(\Theta)}$ is a modified shear modulus, which leads, in the limit case, to a rigid-viscoplastic behavior for the liquid-like state.

The thermal deformation, $\varepsilon^{\Theta}$, is computed considering the evolution the thermal shrinkage during the change of phase, $\varepsilon^{p c}(\Theta)$ as:

$\varepsilon^{\Theta}(\Theta)=\varepsilon^{p c}(\Theta)=\frac{\rho(\Theta)-\rho_{L}}{\rho_{S}}$ 
where $\rho(\Theta)$ is the (temperature dependent) density. Observe that when $\Theta=\Theta_{L}$ the thermal deformation vanishes.

Finally, the model introduces a J2-yield-surface where the (temperature dependent) yield-surface radius $R(q, \Theta)$ vanishes according to the solid fraction evolution, as:

$R(q, \Theta)=f_{S}(\Theta) \sqrt{\frac{2}{3}}\left[\sigma_{0}(\Theta)-q\right]$

This is the generalization of Eq. (24) which enforces a rigidviscoplastic behavior when the material is liquid while restoring the standard elasto-viscoplastic model when the material solidifies.

\subsection{Liquid-like state $\left(\Theta>\Theta_{L}\right)$}

In this case, the material behavior is characterized by a purely viscous (Norton-Hoff) model which can be written as:

$$
\left\{\begin{array}{l}
\nabla \cdot \mathbf{u}=0 \\
\mathrm{~s}=\eta \dot{\varepsilon}^{v p}
\end{array}\right.
$$

According to this model, the deviatoric part of the stress tensor is computed as a function of the visco-plastic strain-rate and the (temperature dependent) viscosity parameter, $\eta(\Theta)$. Eq. (30)-a corresponds to Eq. (20)-a when the material is liquid $(K \rightarrow \infty$, and $\left.e^{\ominus}=0\right)$. Observe that no volumetric deformations are allowed above the liquidus temperature (the material is incompressible) so that Eq. (30)-a represents the continuity equation for mass conservation.

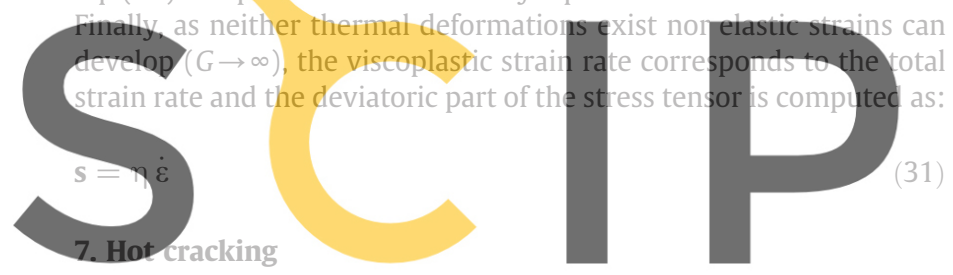

Intergranular liquation and cracking in the weld heat affected zone

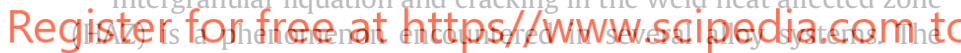
combination of liquid films along grain boundaries and thermal tensile stresses induced by the thermal deformations during the thermal cycles of the SMD process may lead to the formation of irregular cracks.

In the nickel super-alloy 718 the formation of liquid films has been attributed to the liquation of MC-carbides and Laves phase. When the alloy is rapidly heated to a temperature above the binary eutectic temperature, there may be insufficient time for the complete dissolution of the Laves phase and a liquid film is generated to later re-solidify in accordance with the phase diagram requirements. If tensile stresses develop the risk of cracking is high [23].

The prediction of the hot cracking phenomena is a very challenging task from the computational point of view [24]. In fact, it is very difficult to correlate the evolution of the metallurgy with the macro-phenomenological model presented in the previous section. The metallurgical scale is much smaller than the macro scale where the FE mesh is defined. The level of definition of the FE mesh usually adopted for the numerical simulation of the SMD process is insufficient to consider such complex material behavior and the time scale which should be used to study the microstructure kinetics is incompatible with the one used in an industrial simulation. Both temperature gradients, and phase change evolution can be captured only in terms of thermal shrinkage and latent heat release and in many cases the non-linearity induced by such phenomena should require a much finer mesh and time-stepping selection too.

In this work an alternative approach is adopted. The model proposed is based on macroscopic quantities such as strains, stresses and temperature fields (see [12]) which are the natural output of the thermo-mechanical simulation of the SMD process. The proposed methodology consists of computing a damage induced porosity in the mushy zone according to the solid fraction evolution in the temperature range $\Theta_{E} \leq \Theta \leq \Theta_{S}$, that is, between the eutectic temperature, $\Theta_{E}$ (when the Laves solidify at the end of the segregation process) and the solid temperature, $\Theta_{S}$ (when the matrix solidifies). Within this temperature range, the macroscopic model proposed for the mushy phase behavior must account for the shrinkage of the material induced by the phase change. This thermal deformation is volumetric and conventional models such as the one proposed in the previous section do not account for plastic volumetric change because it assumes a von Mises viscoplastic (deviatoric) behavior.

To overcome this, the constitutive model is enhanced with a damage induced porosity behavior including the effects of the drastic volume changes of the phase transformation shrinkage.

A von Mises based visco-plastic model does not pose any restriction to the hydrostatic stress (pressure) limit neither for compression nor for tensile stresses [27]. The objective of the hot-cracking model is to prevent from an excessive (tensile) stress level when the material, still partially liquid, contracts (shrinkage) but all the surrounding phase has solidified.

The present work makes use of a continuum damage model to characterize the porosity formation. In particular, a damage index (hot cracking risk indicator), $d$, is introduced as one scalar internal variable leading to a simple constitutive model which, nevertheless, is able to reproduce the overall non-linear behavior including stiffness degradation and hot tearing phenomena. The constitutive equation used to compute the pressure field, within the temperature range $\Theta_{E} \leq \Theta \leq \Theta_{S}$, results in:

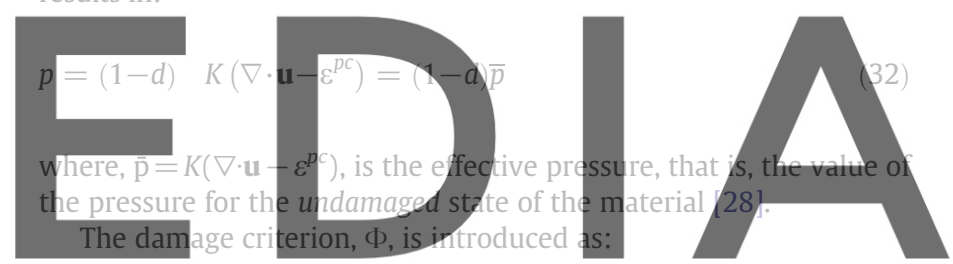

\section{$\Phi(\bar{D}, r)=\bar{p}-r \leq 0$ \\ download the version without the watermark}

Variable $r$ is an internal stress-like variable, which can be interpreted as the current damage threshold, in the sense that its value controls the size of the (monotonically) expanding damage surface: $\dot{r} \geq 0$. The initial value of the damage threshold is $r_{0}=p_{0}$, where $p_{0}$ is the pressure threshold for the undamaged material.

The evolution of the damage bounding surface, for loading, unloading and reloading conditions, is controlled by the Kuhn-Tucker relations:

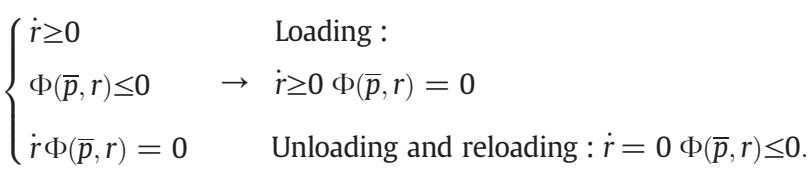

The consistency condition, $\dot{\mathrm{r}} \dot{\Phi}(\overline{\mathrm{p}}, r)=0$, together with Eq. (32) is used to obtain an explicit definition of the current value of the damage threshold in the form [29]:

$r=\max \left\{r_{0}, \max (\bar{p})\right\}$

This given, if the $\overline{\mathrm{p}}>r$, the damage threshold is updated to $r=\overline{\mathrm{p}}$ and the damage index $d=d(r)$ is updated accordingly as:

$d(r)=\frac{r-r_{0}}{r}$.

Observe that the damage variable is only allowed to monotonically increase $\dot{d} \geq 0$ and it can vary within the range $0 \leq d \leq 1$ : hence $d=1$ means $100 \%$ hot-cracking risk. 


\section{Variational formulation}

The solution of the coupled thermo-mechanical problem consists of enforcing the weak forms of either the balance of energy equation or the balance of momentum equation. This means integrating both Eqs. (1) and (18) over the domain $\Omega$ together with the corresponding Dirichlet and Neumann boundaries conditions on $\partial \Omega$.

Consequently, the weak form associated to the energy balance equation, which solves the thermal problem, takes the following expression [6,7]:

$\left(\delta \vartheta, C_{\Theta} \dot{\Theta}+\dot{L}_{p c}\right)+\left(\nabla(\delta \vartheta), k_{\Theta} \nabla \Theta\right)=(\delta \vartheta, \dot{R})+\left(\delta \vartheta, q_{\text {rad }}+q_{\text {conv }}\right)_{\partial \Omega} \quad \forall \delta \vartheta$

where $\delta \vartheta$ are the test functions associated to the temperature field, $\Theta$, while $(\cdot, \cdot)$ denotes the inner product in $L^{2}(\Omega)$.

It is important to remark that the SMD process induces extremely high temperature gradients along the welding path in the heataffected zone. From the numerical point-of-view such temperature gradients can provoke unrealistic temperature overshots and undershots especially if coarse meshes and large time-steps are used. In this work, the problem has been avoided adopting a nodal (Lobatto) integration rule, which stabilizes the solution introducing a small numerical diffusivity into the heat conduction problem [6,7]

The weak form of the balance of momentum equation can be stated as:

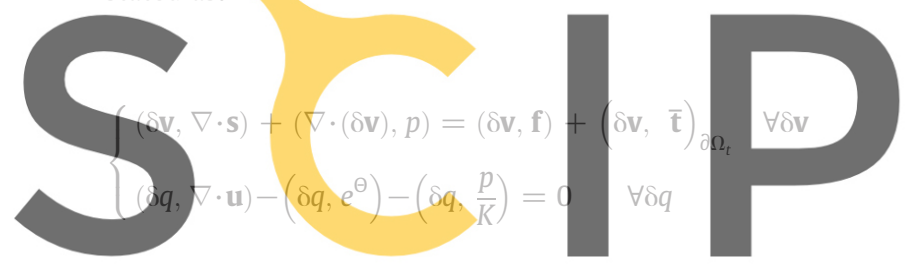

$(38$

\section{where ov and oq are the variations of the displacement and pressure ister fołcfree at https//www.scipedia.com to} Observe that both the J2-plasticity (solid model) and the purely viscous behavior (liquid model) generate only deviatoric deformations (isochoric response) leading to possible locking problems. Recent works of the authors in the field of incompressibility in solid mechanics [16-19] show the necessity of an ad-hoc finite element technology. The mixed variational formulation proposed, uses linear displacements and pressure interpolations, leading to robust triangular or tetrahedral elements suitable for large-scale computation of constrained media problems. The orthogonal sub-grid scale approach $[20,21]$ is assumed as an attractive alternative to circumvent the Babuska-Brezzi stability condition [22]. When meshing with hexahedral elements, the B-bar method or the equivalent Q1/P0 element technology is an alternative formulation to deal with this problem.

\section{Time integration of the coupled problem}

The numerical solution of the coupled thermo-mechanical problem (Eqs. (37) and (38)) involves the transformation of an infinite dimensional transient system into a sequence of discrete non-linear algebraic problems. This can be achieved by means of a Galerkin finite element projection and a time-marching scheme for the advancement of the primary nodal variables, displacements, pressure and temperatures, together with a return mapping algorithm to update of the internal variables.

With regard to the time stepping scheme different strategies are possible, but they can be grouped in two categories: simultaneous (monolithic) solution and staggered (block-iterative or fractionalstep) time-stepping algorithms.
In this work a staggered solution has been chosen. A product formula algorithm has been introduced leading to a time-integration scheme in which the two sub-problems (thermal and mechanical) are solved sequentially, within the framework of classical fractional step methods [6,7]. As a result, the original coupled problem is split into two smaller and typically symmetric partitions allowing the use of any integration technique originally developed for the uncoupled subproblems.

The final result is an accurate, efficient and robust numerical strategy, which allows the numerical simulation of large coupled thermo-mechanical problems such as the multi-pass welding and the SMD processes.

\section{FE modeling of the SMD process}

The shaped metal deposition (SMD) process is a welding technology in which the filler material is melted and deposited layer-by-layer. From the numerical point of view, the simulation of the SMD process requires an ad-hoc finite element activation technology able to reproduce the deposition of the melted material along the welding path. In the literature two different approaches have been used to simulate the multi-pass welding, referred to as quiet elements and born-dead elements techniques [1-5].

The first approach considers that all the elements of the mesh defining the successive welding layers, which will be deposited during the process simulation, are included into the initial computational model. These elements are made passive (quiet) by setting material properties which do not affect the rest of the model (penalization).

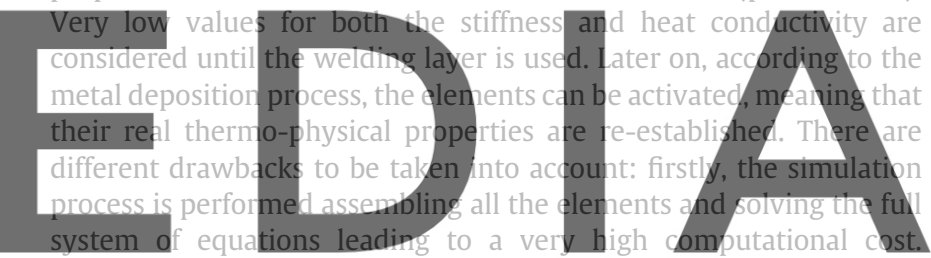
Secondly, the penalization of the material properties of the passive elements induces an ill-conditioning of the solution matrix which may

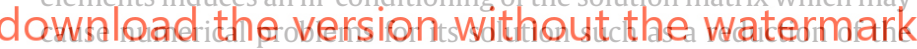
convergence ratio of iterative solvers. Finally, fictitious strains and temperature gradients in the quiet elements are accumulated and they will be transformed into spurious stresses and heat fluxes when these elements are activated. On the other hand, the implementation is straightforward.

The simulation strategy adopted in this work to deal with the SMD process consists of an ad-hoc activation process based on the so called born-dead elements technique. This activation strategy classifies the elements defined in the original finite element mesh into: active, inactive and activated elements. The active elements (e.g. the mesh which define the base material) are computed and assembled into the global matrix. On the other hand, the inactive elements, such as the entire discretized domain where the welding path is defined, are not assumed as part of the model: they have been generated but do not play any role in the computational model. At each time-step a number of elements (activated elements) are switched on according to the deposition of the filler material along the welding-path defined by the user. Only active and activated elements are assembled into the solution model.

The activation technique uses a control volume defined by the cross section of the SMD layer and the movement of the arc in the welding direction, $d=v_{M D} \cdot \Delta t$, defined in terms of the welding speed, $v_{M D}$ and the current time-step, $\Delta t$ (see Fig. 3 ). All the elements belonging to this domain are identified by a searching algorithm, their status is switched from inactive to activated elements and they are added to the model. Fig. 3 shows the control volume defined to activate the corresponding elements on the welding path for a 3D analysis. Mass conservation requires that the volume of the SMD 


\section{a}
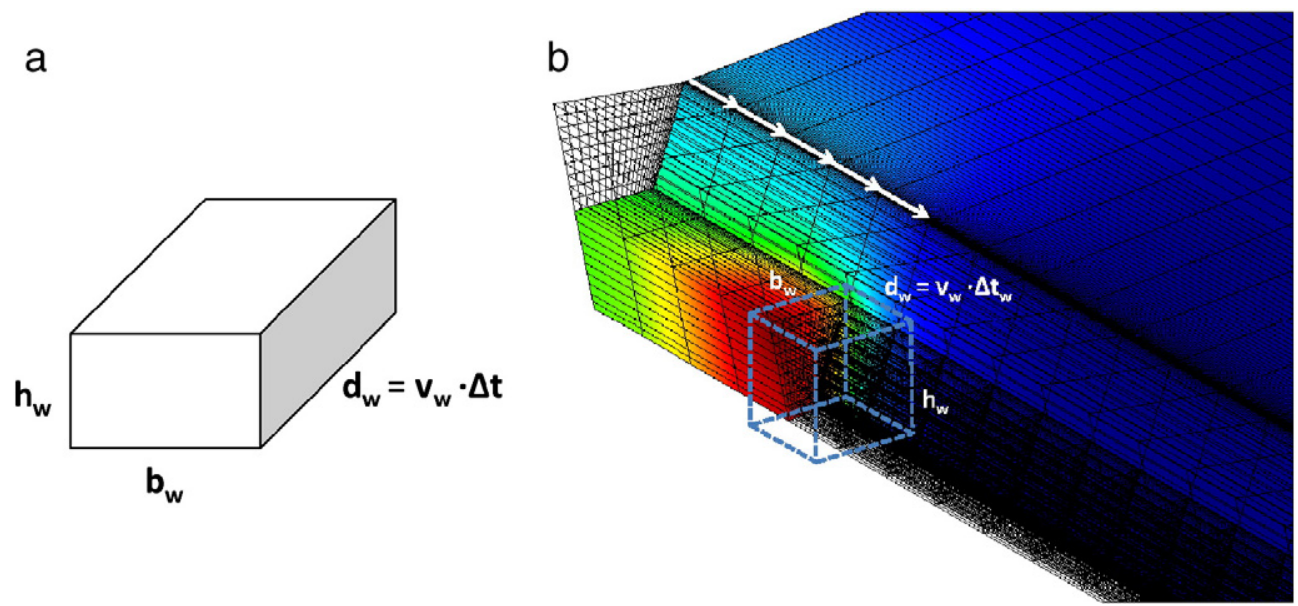

Fig. 4. Activation strategy: a) Control volume defined for the activation procedure; b) Control volume used for an activation process.

Table 1

Thermal physical properties and mechanical properties of AISI 304.

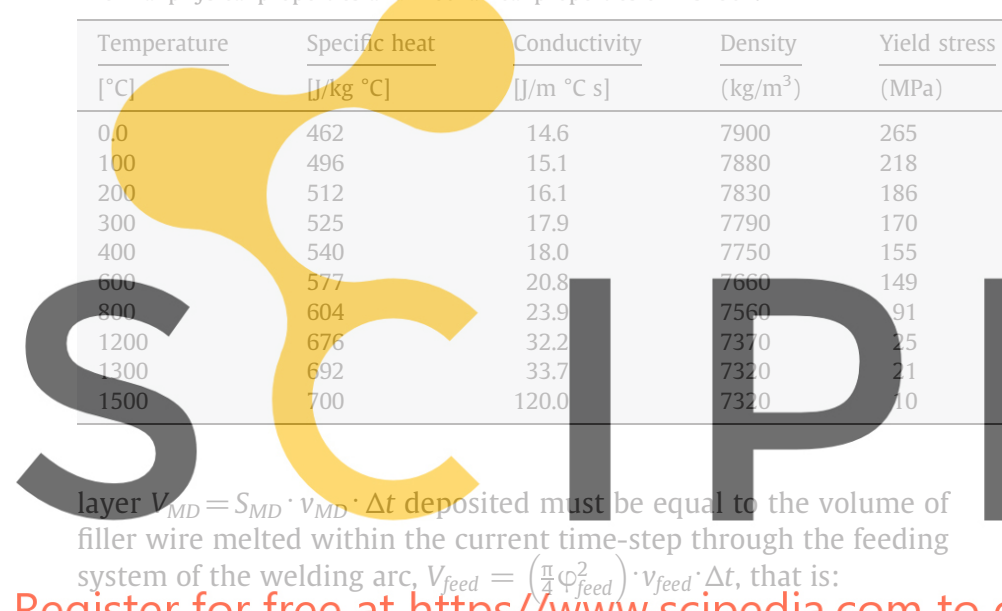

Register for free at https//wWW.scipedia.com to

$V_{M D}=V_{\text {feed }}$

where $\varphi_{\text {feed }}$ and $v_{\text {feed }}$ are the diameter of the filler wire and the feeding speed, respectively. From this, the cross section, $S_{M D}$, of each SMD layer deposited according to the welding speed of the arc and the feeding settings, is computed as:

$S_{M D}=\left(\frac{\pi}{4} \varphi_{\text {feed }}^{2}\right) \cdot \frac{v_{\text {feed }}}{v_{M D}}$

The advantage of this activation technique is the possibility of defining the computational mesh independently from the welding path. At each time-step of the simulation the profile of the solution matrix, as well as the number of equations, is changing according to the activation process. Compared to the quiet elements technology, more accurate results can be achieved even if this more sophisticated activation methodology is more time consuming.

Table 2

Stainless steel pipe: welding process parameters.

\begin{tabular}{llll}
\hline Test & Current $[\mathbf{A}]$ & Voltage $[\mathbf{V}]$ & Speed $[\mathbf{m m} / \mathbf{m i n}]$ \\
\hline$\# 1$ & 140 & 9.5 & 80 \\
$\# 2$ & 140 & 9.5 & 800 \\
$\# 3$ & 140 & 9.5 & 8000 \\
$\# 4$ & 140 & 9.5 & $\infty$ (instant activ.) \\
\hline
\end{tabular}

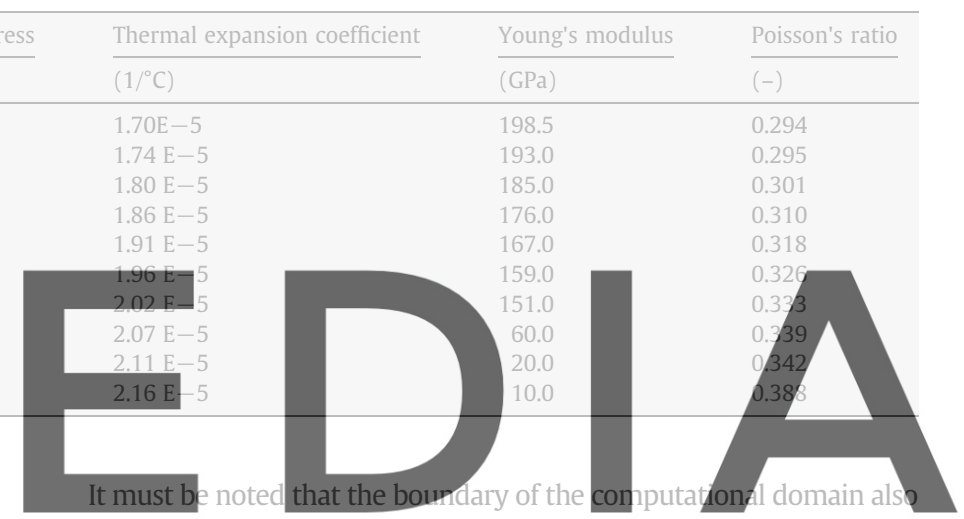

changes due to the activation process, requiring a specific searching strategy to update the active surface at each time-step of the numerical

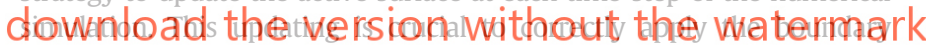
conditions such as the heat radiation and heat convection fluxes according to the activation process.

unnfortunately, the SIVID process that we are trying to simulate should require a mesh for the base material being independent from the one defined for the filler material during the deposition. This way, the dilatation of the filler material, passing through the welding-arc in the heating phase, could take place independently (till the solidification) from the deformation of the base material. This is the best option that one could think of, but unfortunately it requires contact elements

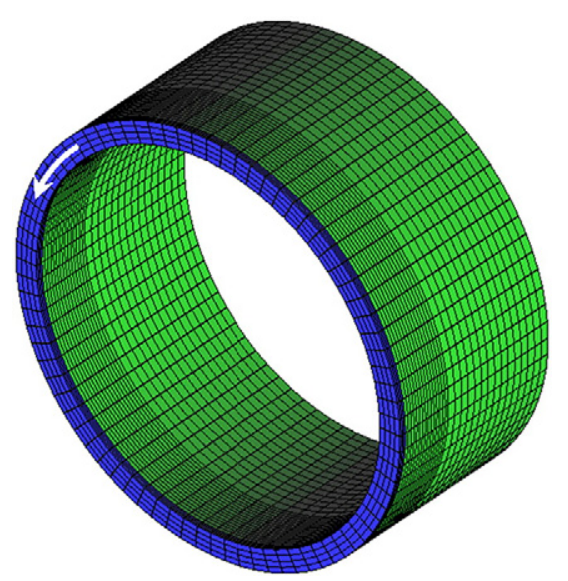

Fig. 5. 3D model and finite element mesh used for the numerical simulation of the welding process of the stainless steel pipe. 

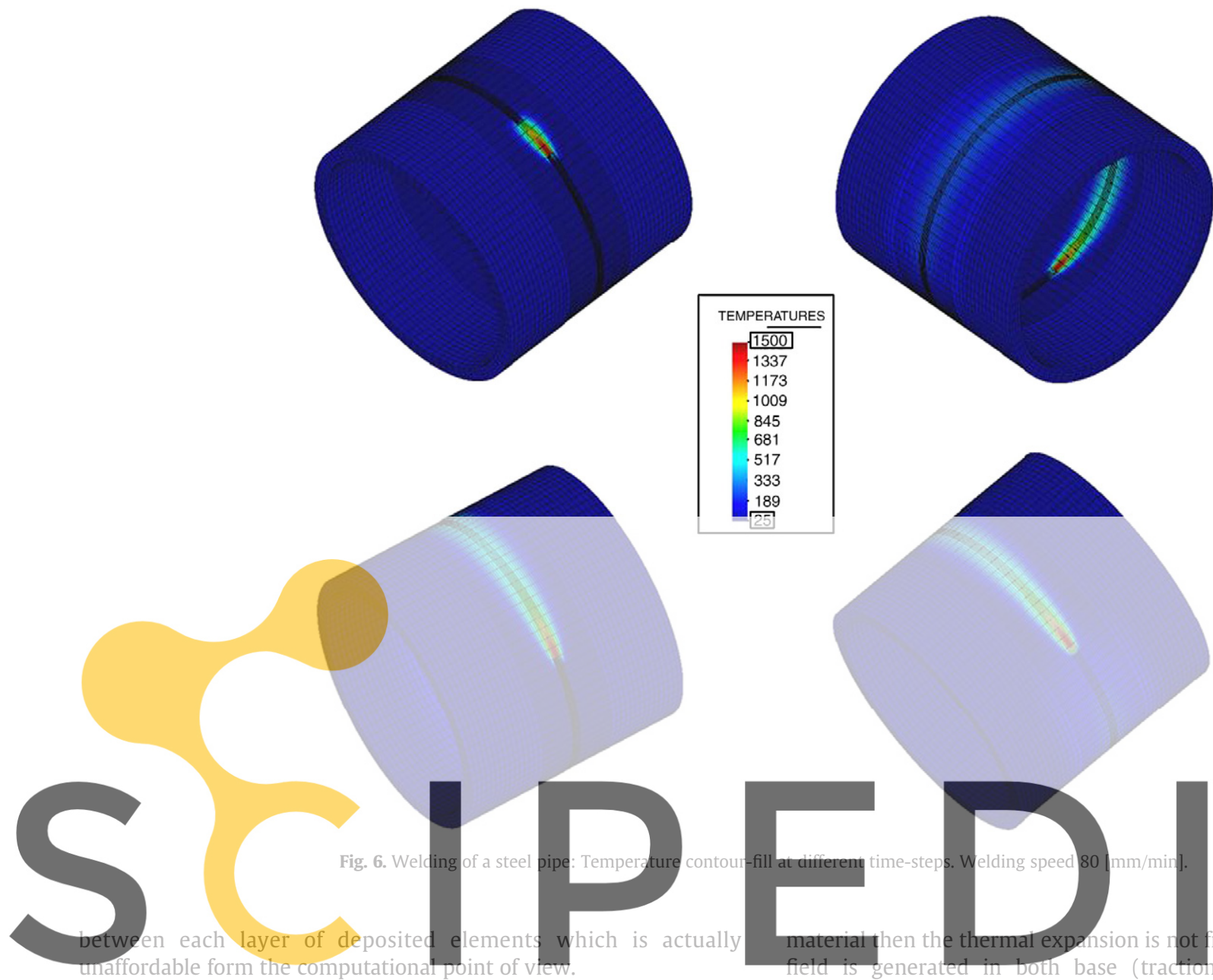

The solution proposed in this work considers one unique mesh so

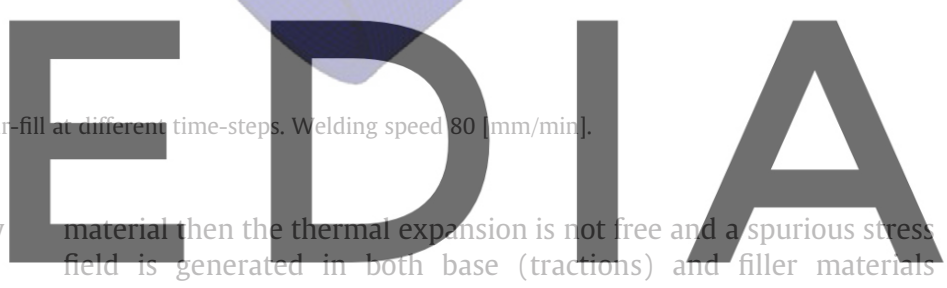

(compressions). Hence, to get an accurate mechanical response, it is

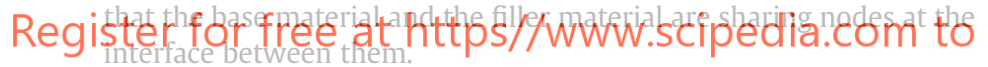

During the SMD simulation, each new activated element is born with an initial displacement field, $\mathbf{u}_{0}$, induced by the movement of the nodes shared with the pre-existing active elements. At the instant of the activation, this initial displacement field, if it is not removed from the computation, transforms into a spurious pre-stress field which pollutes the solution. In the industrial process, the thermal expansion of the filler material can develop without any constraining. Numerically, if the nodes of the filler material are shared with the base

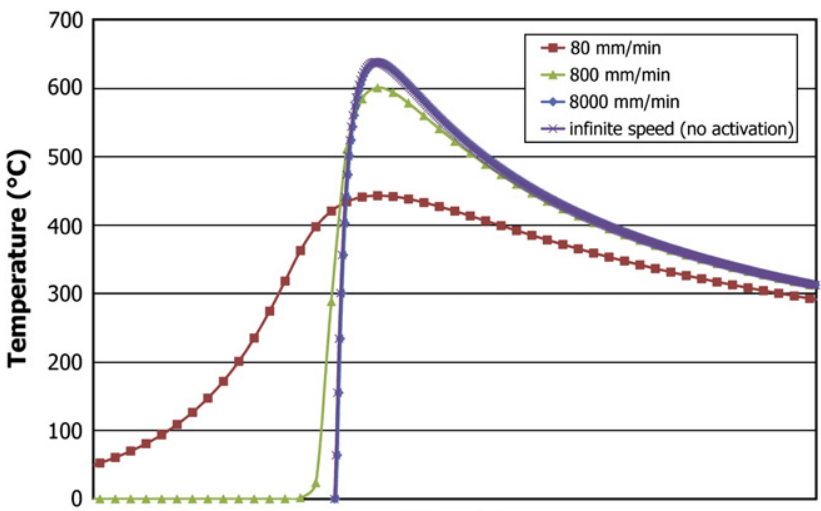

Time (s)

Fig. 7. Welding of a steel pipe: Temperature profiles at different welding speed: 80,800 , $8000[\mathrm{~mm} / \mathrm{min}]$ and infinite speed (no activation).

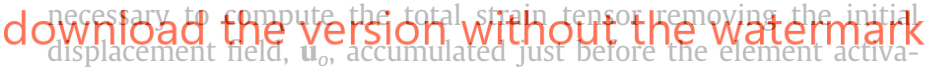
tion, as:

$\varepsilon=\nabla^{S}\left(\mathbf{u}-\mathbf{u}_{0}\right)$

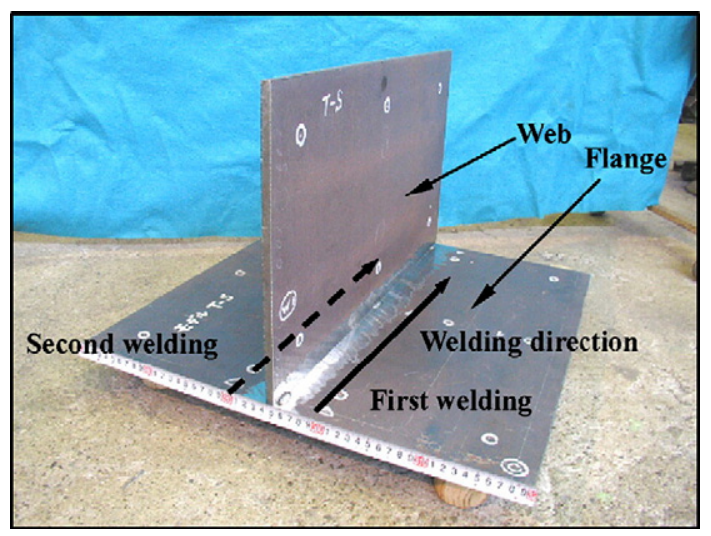

Fig. 8. T-joint experimental setting.

Table 3

T-joint: welding parameters.

\begin{tabular}{lllll}
\hline Shielding gas & Wire & Current $[\mathrm{A}]$ & Voltage $[\mathrm{V}]$ & Speed $[\mathrm{mm} / \mathrm{min}]$ \\
\hline $\mathrm{CO}_{2}$ & DW100V & 270 & 29 & 400 \\
\hline
\end{tabular}


Table 4

T-joint: thermal and mechanical temperature-dependent properties.
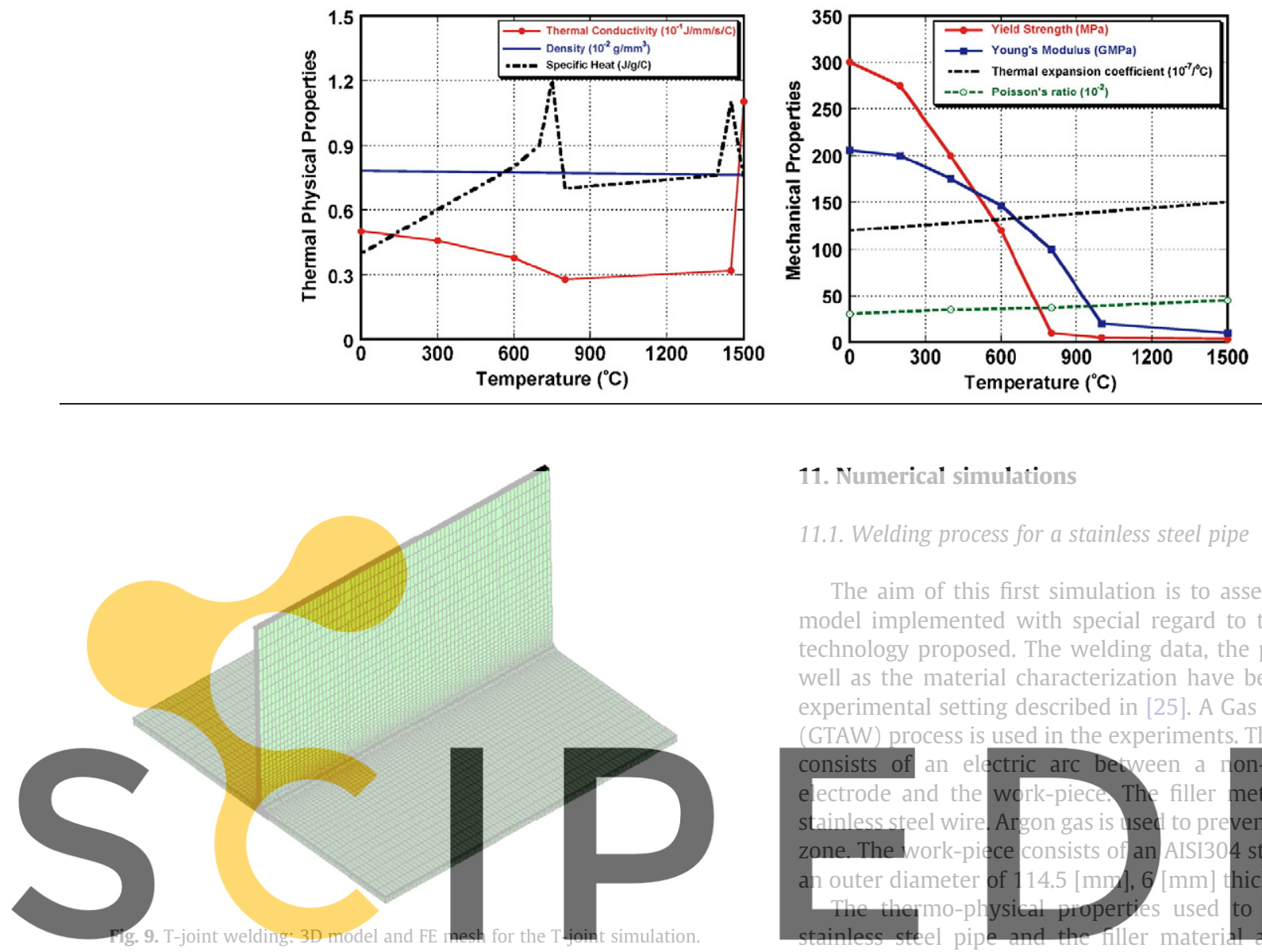

11. Numerical simulations

11.1. Welding process for a stainless steel pipe

The aim of this first simulation is to assess the accuracy of the model implemented with special regard to the element activation technology proposed. The welding data, the process parameters, as well as the material characterization have been obtained from the experimental setting described in [25]. A Gas Tungsten Arc Welding (GTAW) process is used in the experiments. This welding technology

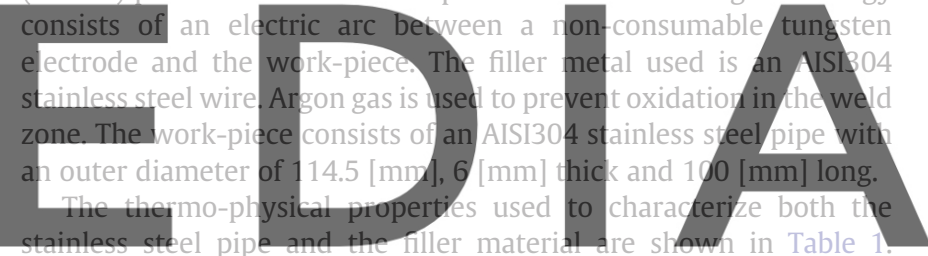

Different analyses have been performed considering the same energy Register for free at https//www.scipedia.com to dinut but differentwelding speeds s seithable â. The efficiency of the

For symmetry reasons, a 3D model of one half of the pipe to be jointed as well as half of the filler material deposited has been considered for
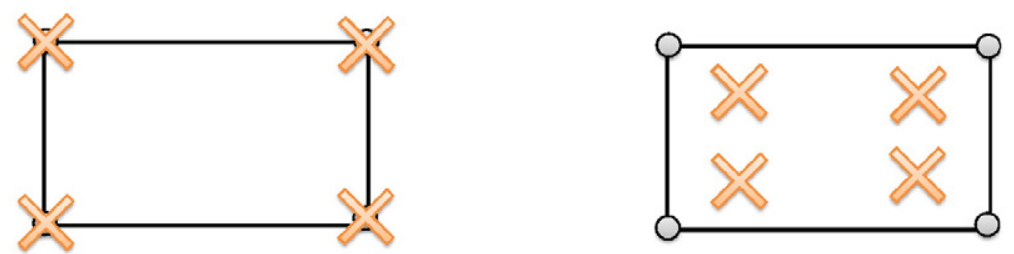

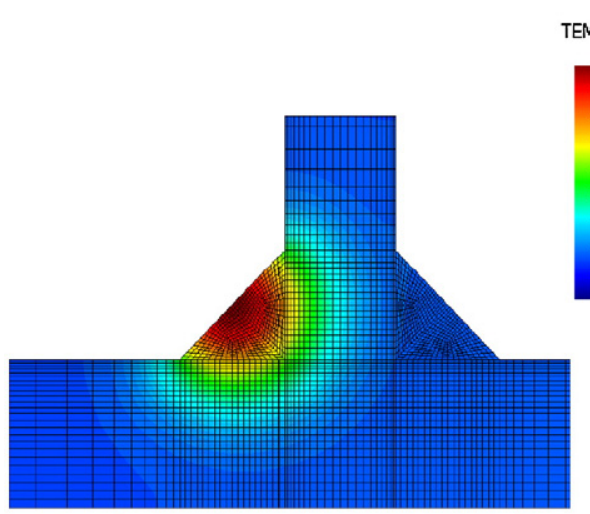

a

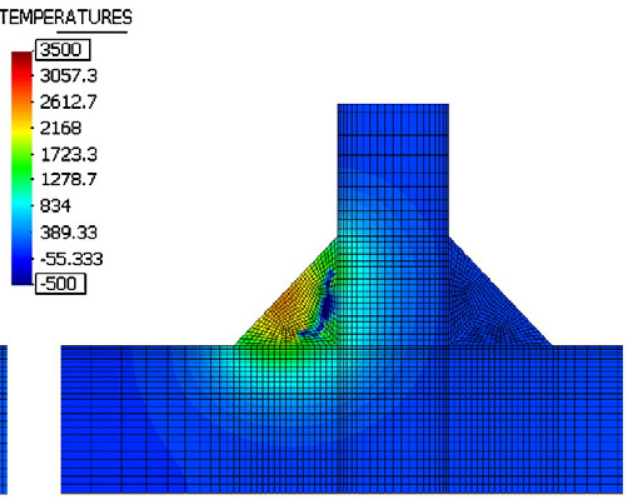

b

Fig. 10. T-joint welding: temperature field in a cross section using: a) Close (Lobatto) integration rule; and b) Open (Gauss) integration rule. 


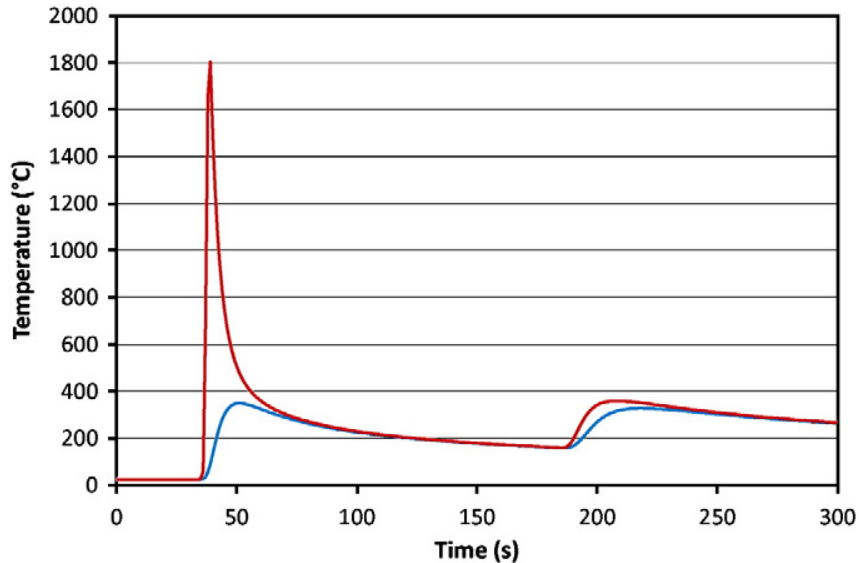

Fig. 11. T-joint welding: temperature evolution at two different locations.

the numerical simulation model. A finite element mesh with 10.880 hexahedrical elements and 14.000 nodes (see Fig. 5) has been used.

The first simulation has been carried out considering an instantaneous activation of all the elements which correspond to the filler material. This hypothesis is equivalent to an infinite welding speed. Moreover, three analyses have been performed using the activation technology proposed in this work, assuming different welding speeds: 80,800 and 8000 [mm/min], respectively. Fig. 6 shows the temperature contour-fill at different time-steps of the simulation for a welding speed of $80[\mathrm{~mm} / \mathrm{min}]$.

Fig. 7 shows the resulting temperature evolution curves at the same location of the thermo-couple for each welding speed considered. It is important to remark that the curve obtained for $8000[\mathrm{~mm} / \mathrm{min}]$ exactly matches the one obtained considering an infinite welding speed. This means that the activation technology proposed is able to introduce into the model exactly the same energy input which correspond to an instantaneous activation where all the elements belonging to the filler material are active from the beginning of the simulation and the corresponding energy input assigned.

This test proves that at lower welding speed, it is really necessary to adopt a numerical strategy to simulate the metal deposition stepby-step. The Péclet number $P e=\frac{h v}{\alpha}$, can be used to decide when it is mandatory to consider the activation strategy. The Péclet number is a dimensionless quantity that compares thermal advection (for SMD case $v$ is the welding speed and $h$ is the characteristic length of the problem, e.g. the thickness of the pipe) with respect to thermal diffusivity, $\alpha=k_{\Theta} / C_{\Theta}$ (where $k_{\Theta}$ is the thermal conductivity and $C_{\Theta}$ the

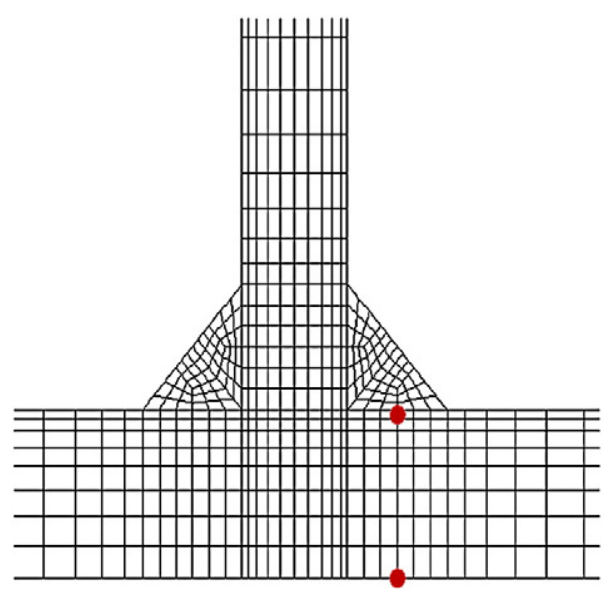

Fig. 12. T-joint welding: locations of the thermo-couples.

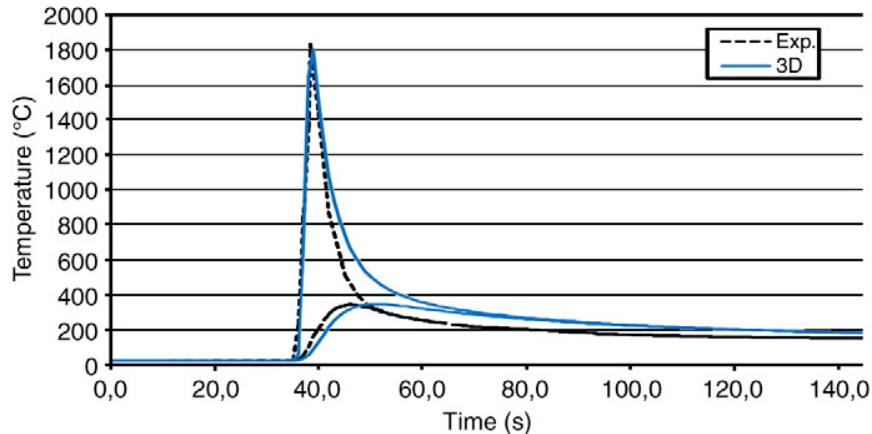

Fig. 13. T-joint welding: Temperature comparison with the experimental result.

heat capacity). Substituting the corresponding data it is possible to conclude that for:

- $P e>50$. The activation strategy is not necessary: instantaneous activation process gives an accurate response;

- $6<P e<50$. In this range the instantaneous activation process shows an error lower than $15 \%$.

- $P e<6$. It is necessary to model the SMD including the element activation process according to the welding speed.

\subsection{Double-pass welding for a T-joint}

The second FE simulation proposed, consists of a fully coupled thermo-mechanical analysis showing the accuracy of the numerical model proposed in terms of both thermal and mechanical model.

The process consists of a fillet-welded T-joint where two welding passes, one at each side, are performed to join the flange to the web.

a

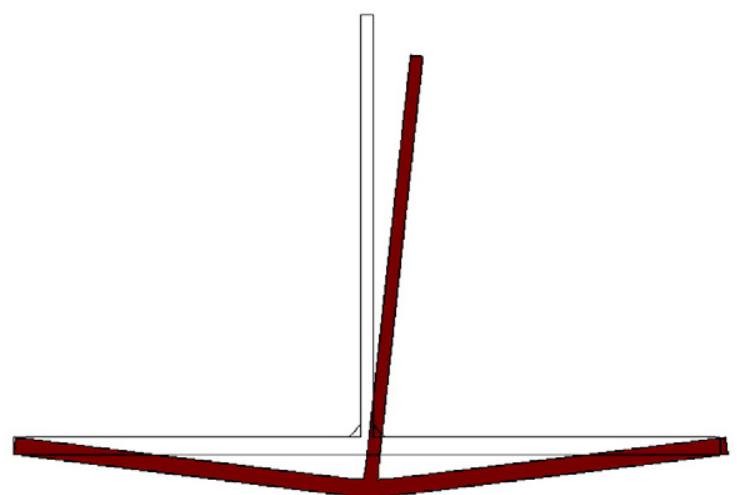

b

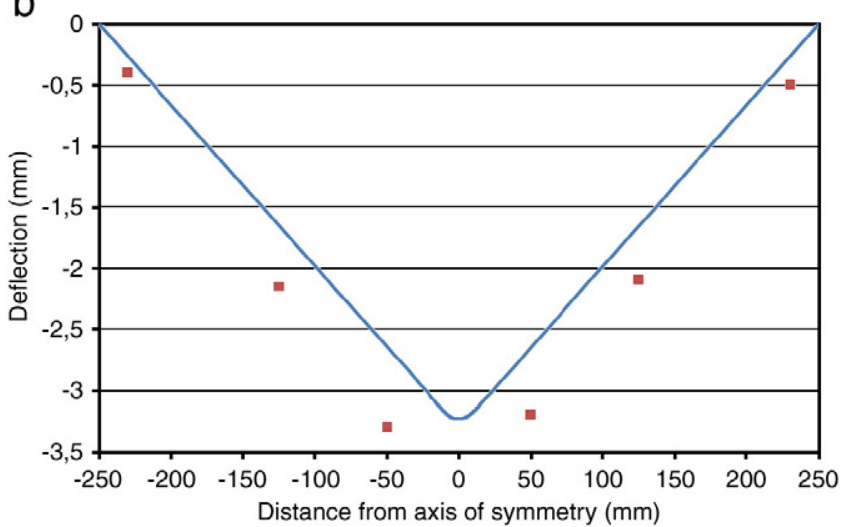

Fig. 14. T-joint welding : a) Deformed shape of the T-joint; b) Vertical deflections at 6 different locations of the transversal section along the flange: comparison between numerical and experimental results. 

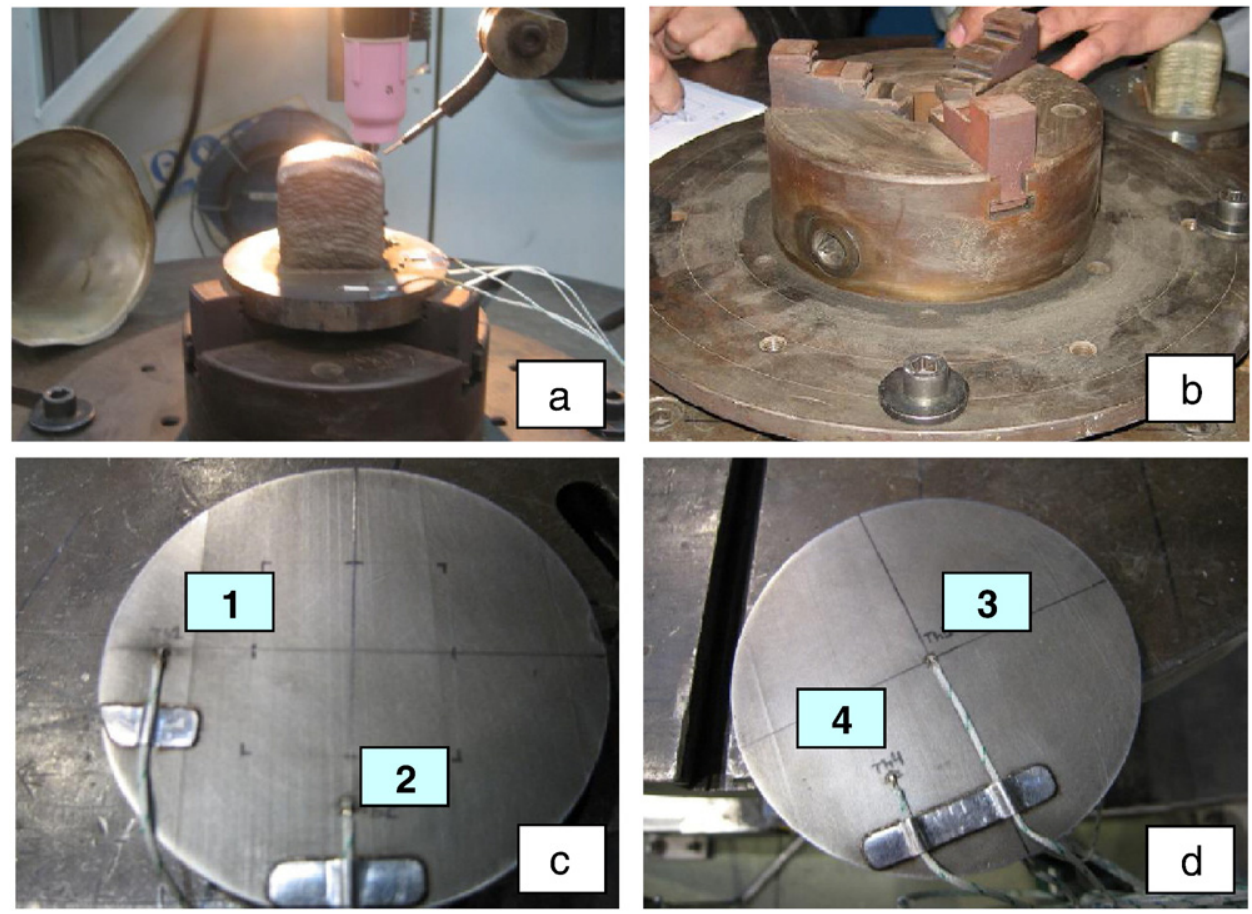

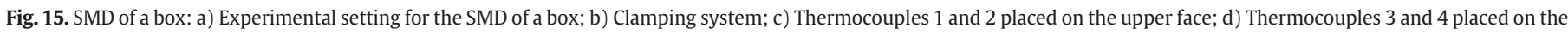
lower face.

The flange is $500[\mathrm{~mm}]$ long, $500[\mathrm{~mm}]$ wide and 12 [mm] thick; the web is $500[\mathrm{~mm}]$ long, $300[\mathrm{~mm}]$ high and $9[\mathrm{~mm}]$ thick. The material used in this test is a ship-building steel SM400A. The welding process is performed using a $\mathrm{CO}_{2}$ gas arc welding. The experimental settings as well as the thermo-physical properties are taken from [26]. The experimental model is shown in Fig. 8. The welding process parameters are detailed in Table 3. Temperature dependent thermomechanical properties are presented in Table 4.

The thermal model considers the latent heat release during the phase change with the melting point at $1350{ }^{\circ} \mathrm{C}$. Both convection and radiation heat fluxes have been assumed. The mechanical model uses an elasto-plastic constitutive law with both isotropic hardening and thermal softening according to the temperature dependent evolution of the yield surface defined by the elastic limit in Table 4 . The thermal shrinkage set during the phase change is of 3\%. Isostatic clamping of the flange is assumed to guarantee that uniform thermal deformations do not induce any stress field.
The 3D model, consisting of 78.300 Q1P0 hexahedral elements and 88.236 nodes, is presented in Fig. 9. The time-step used in the analysis is of $1[\mathrm{~s}]$, allowing for the activation of one element in the direction of the welding path at each time-increment.

Fig. 10 shows the temperature distribution using two different integration rules: Fig. 10a shows the close (Lobatto) integration rule and Fig. 10b shows the open (Gauss) integration rule, respectively. It can be observed in those figures how the pronounced overshoots and undershoots in the temperature field obtained by using a standard open rule can be easily smoothed when adopting the more diffusive close integration rule.

Fig. 11 illustrates the temperature evolution at the two different locations of the thermo-couples shown in Fig. 12, the first one at 1 [mm] from the SMD and the second one on the back-side of the flange. Note that the marked temperature peak affects only the material located very close to the welding. Fig. 13 compares the numerical results corresponding to the first pass with the experimental data

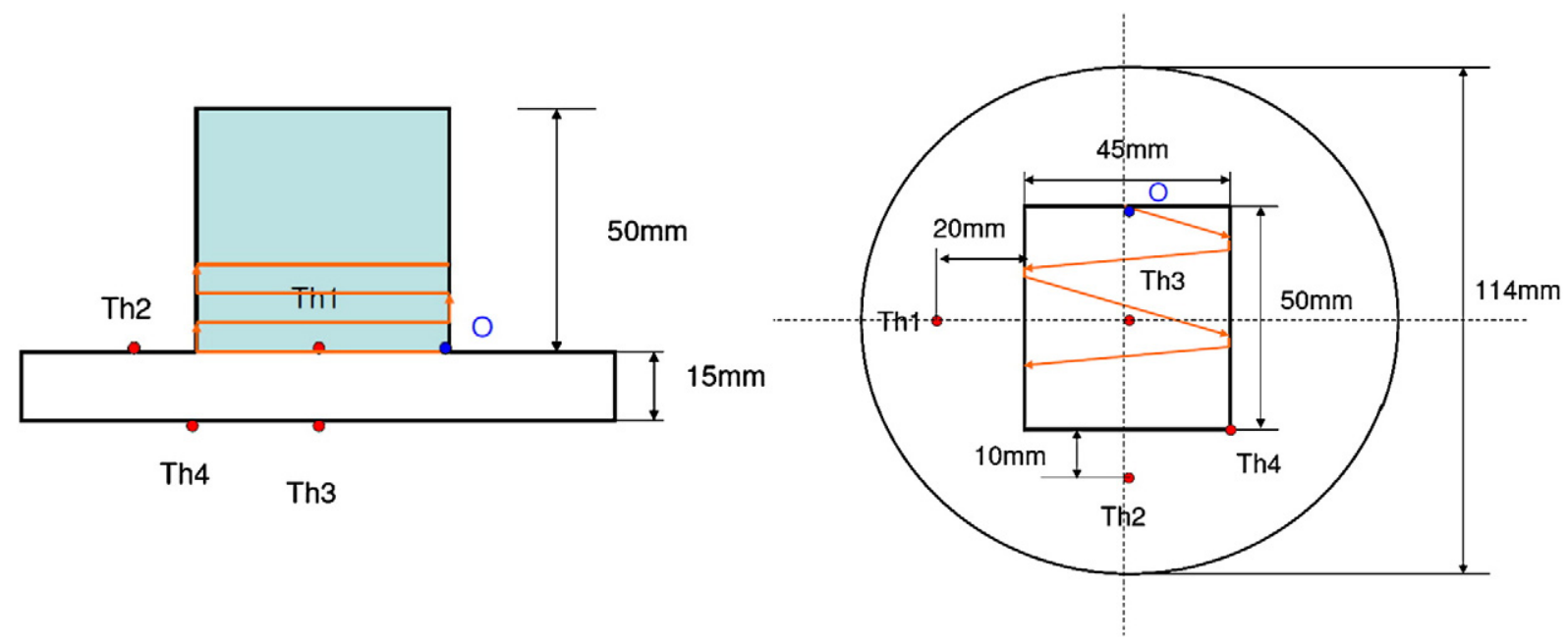

Fig. 16. SMD of a box: Geometry model and thermo-couples locations. 
a

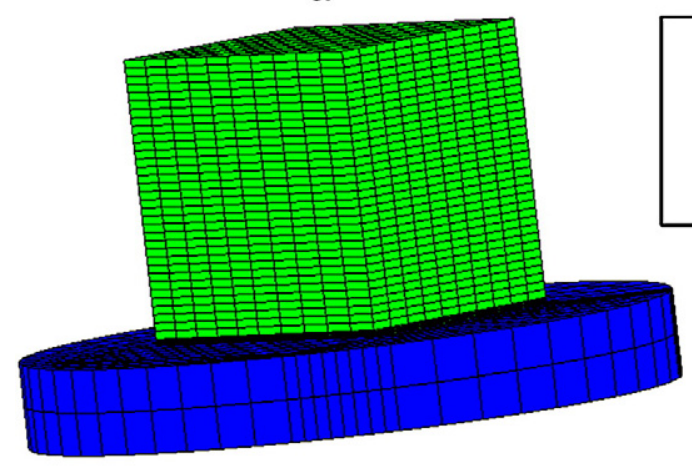

b

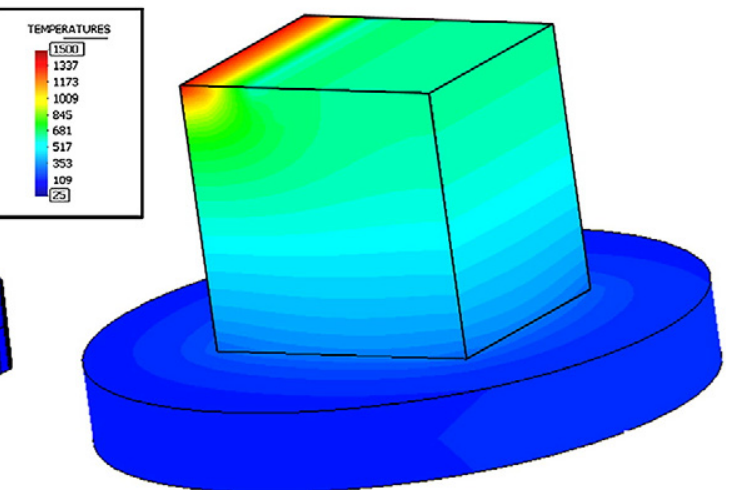

Fig. 17. SMD of a box: a) FE mesh used in the analysis; b) Temperature contour-fill at the end of the SMD process.

available in [26]. The agreement is remarkably good. Fig. 12 shows an asymptotic value for the temperature field for both the numerical and the experimental results. This is just because the cooling phase in the simulation should be much longer to let the temperature decrease to the room value. Unfortunately, the cooling phase is governed by the environment heat transfer coefficient which is a quite small parameter. As a result the heat evacuated through the boundaries is not enough to cool down the T-join as fast as expected.

The accuracy of the mechanical analysis is assessed by comparing the angular distortions of the flange with the experimental measures. Fig. 14a shows the distorted shape of the T-joint obtained with the FE analysis. This shape is not symmetric due to the different plastic deformation field induced by the temperature field. Fig. 14b shows the comparison between the vertical deflections at 6 different locations of the transversal section along the flange. The agreement is very good also in this case.

\subsection{Shaped metal deposition of a box}

To validate and calibrate the numerical model presented when a SMD process is considered, a large number of experimental tests have been carried out at ITP Industry (Industria de Turbo Propulsores, SA, Spain).

A Gas Tungsten Arc Welding (GTAW) is used to melt the filler material. The experimental setting is shown in Fig. 15a. Both the base material and the filler material (wire) is nickel super-alloy 718 . The clamping system used to retain the plate has been designed to guarantee that uniform thermal distortions can develop without inducing any stress fields (see Fig. 15b).The temperature evolution is recorded by four thermocouples as shown in Fig. 15c and d.

The geometrical modeling consists of two different parts: the base plate and the metal deposition material, which is activated according to the given welding path (see Fig. 16). The base plate has a cylindrical
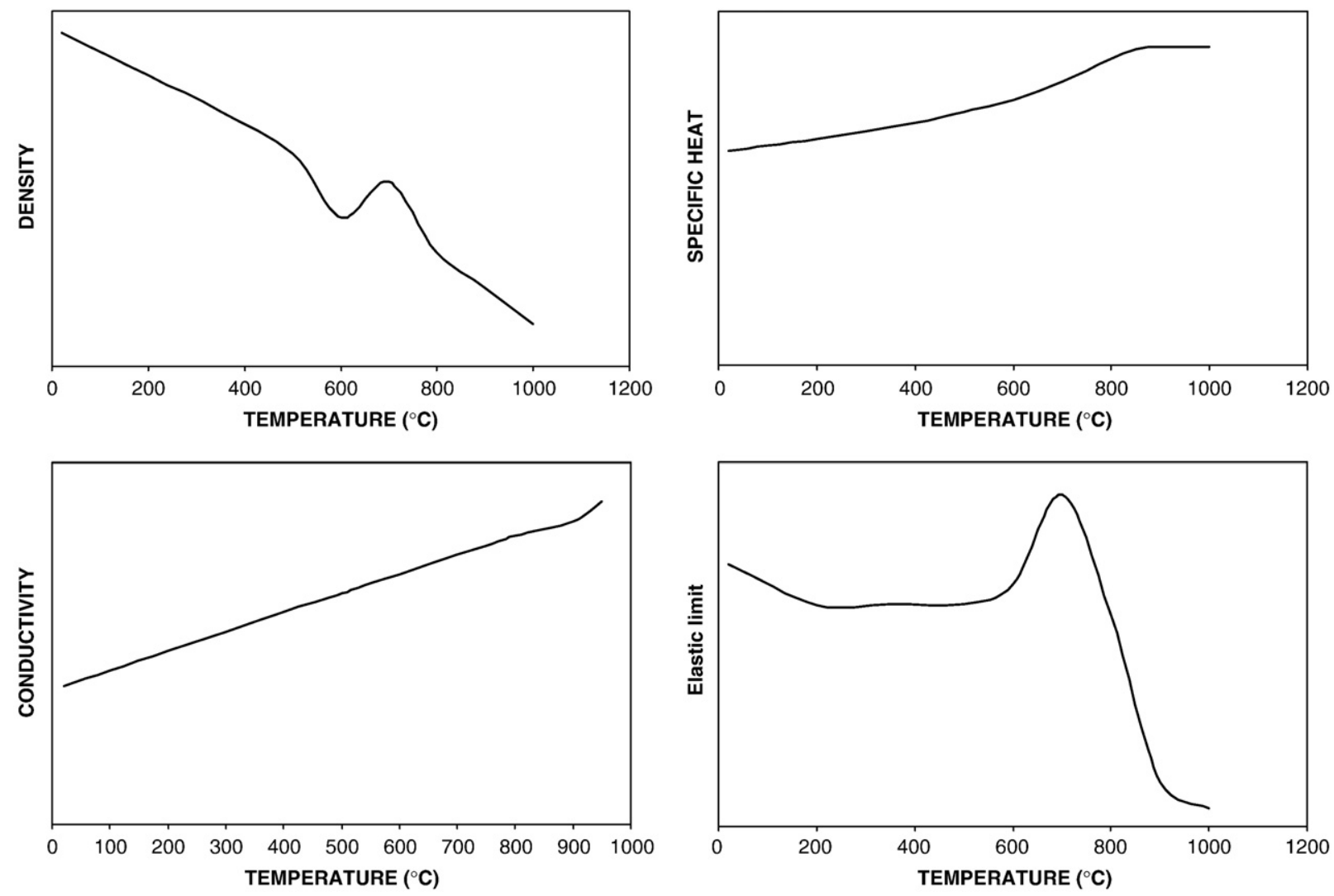

Fig. 18. Temperature dependent material properties for nickel super-alloy 718. 


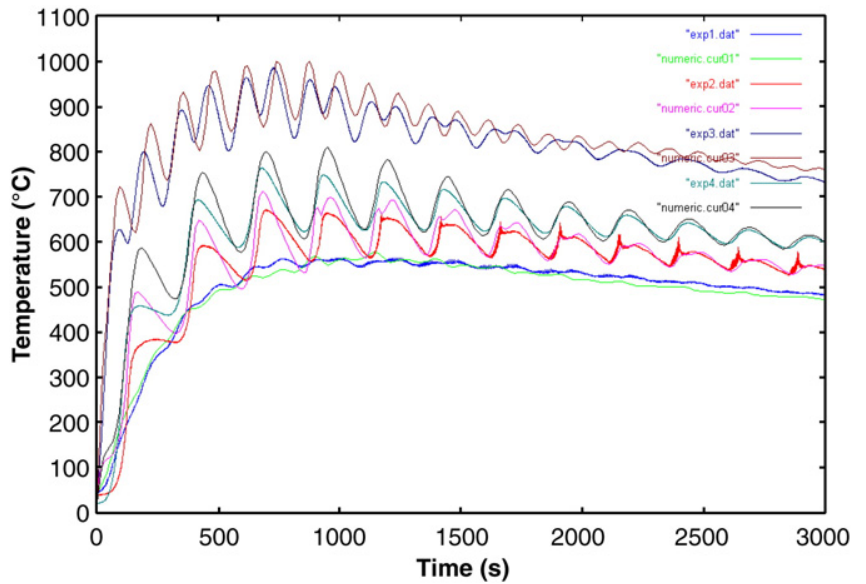

Fig. 19. SMD of a box: Comparison between numerical and experimental results at the 4 thermocouples locations.

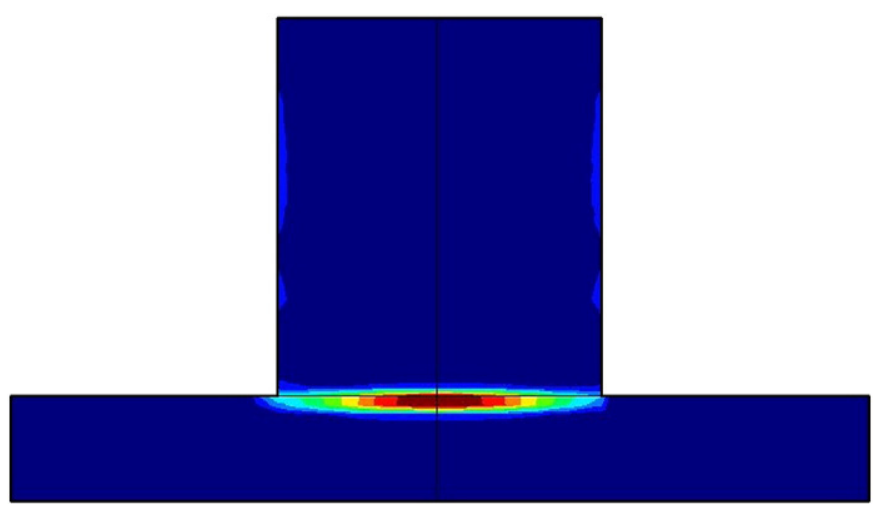

Fig. 20. SMD of a box: Hot cracking risk indicator.

shape $15[\mathrm{~mm}]$ high and with a diameter of $114[\mathrm{~mm}]$ while the SMD area is defined by a rectangular shape $45[\mathrm{~mm}]$ long and $50[\mathrm{~mm}]$ wide which will grow according to the SMD process up to the final height of $50[\mathrm{~mm}]$.

A finite element mesh for the 3D model consists of 6.114 hexahedral elements and 6.496 nodes (see Fig. 17a). The analysis considers 10 timesteps for each layer activated, that is, 400 time-steps.

The material properties used for this analysis are confidential. The material behavior modeled is nickel super-alloy 718 in all the temperature range varying from room to the melting temperature.
Fig. 18 shows the temperature dependency of some of the most characteristic material properties within the temperature range considered in this simulation. The thermal model considers a latent heat release during the phase change with the melting point at $1335^{\circ} \mathrm{C}$. Both convection and radiation heat fluxes have been taken into account in this analysis.

Fig. 17b shows the temperature contour-fill at the end of the deposition, just before the cooling phase.

The comparison between numerical and experimental results is presented in Fig. 19 where it is possible to appreciate the good agreement achieved, assessing the accuracy of the numerical model proposed.

Fig. 20 shows the hot cracking risk indicator computed according to the damage model proposed in this work.

\subsection{0 layer SMD process}

The second SMD test case is intended to assess the accuracy of proposed method when a fully coupled thermo-mechanical analysis is applied to predict both the temperature evolution and the distortion of the structure.

The experimental setting is shown in Fig. 21a: it consists of a rectangular base plate $275[\mathrm{~mm}]$ long, $100[\mathrm{~mm}]$ wide and $12[\mathrm{~mm}]$ thick. The SMD process consists of 2 pre-heating passes (no feeding material is used) followed by the deposition of 10 layers of material.

An isostatic clamping of the base plate is assumed to avoid any stress concentration induced by the thermal deformation of the plate (see Fig. 21b).

The material used is nickel super-alloy 718. The welding arc and welding efficiency are set out as in the previous case.

The finite element model consists of 54.862 Q1/P0 hexahedral elements and 34,012 nodes (Fig. 22b).

Fig. 23b-d compares the temperature evolution at the different thermocouple locations as shown in Fig. 23a.

Fig. 24 shows the Z-displacement field achieved by the analysis after the cooling phase. The comparison between experiment and numerical results for a transversal and a longitudinal section are presented in Figs. 25 and 26, respectively [10]. It is possible to observe how numerical results underestimate the deflection. One possible reason is the kinematic adopted. Most probably when using a largestrain formulation the results will look better but on the other hand this formulation is too expensive from the computational point of view when running industrial cases.

Finally, Fig. 27 compares the residual stress field at three different locations of the strain-gauges. Hole drilling method has been used to obtain the residual stresses at the different locations. J2 von Mises a

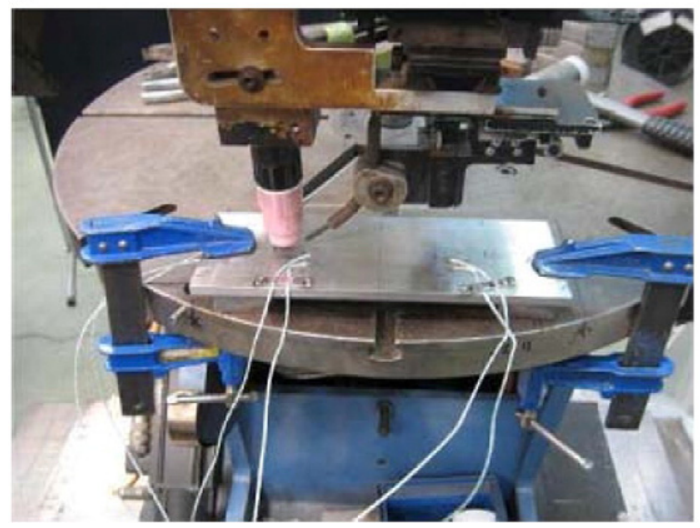

b

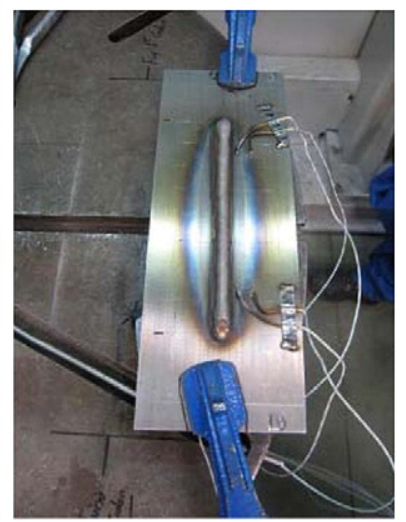

Fig. 21. 10-Layers SMD test: a) Experimental setting for the 10 layers SMD test; b) Clamping system. 
a

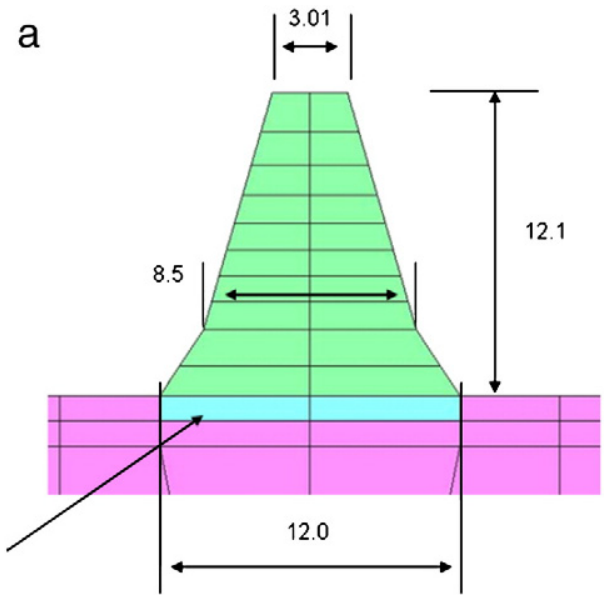

Fig. 22. 10-Layers SMD test: a) Cross section for the 10-layers SMD process; b) FE mesh used for the numerical simulation of the 10-layers SMD process.

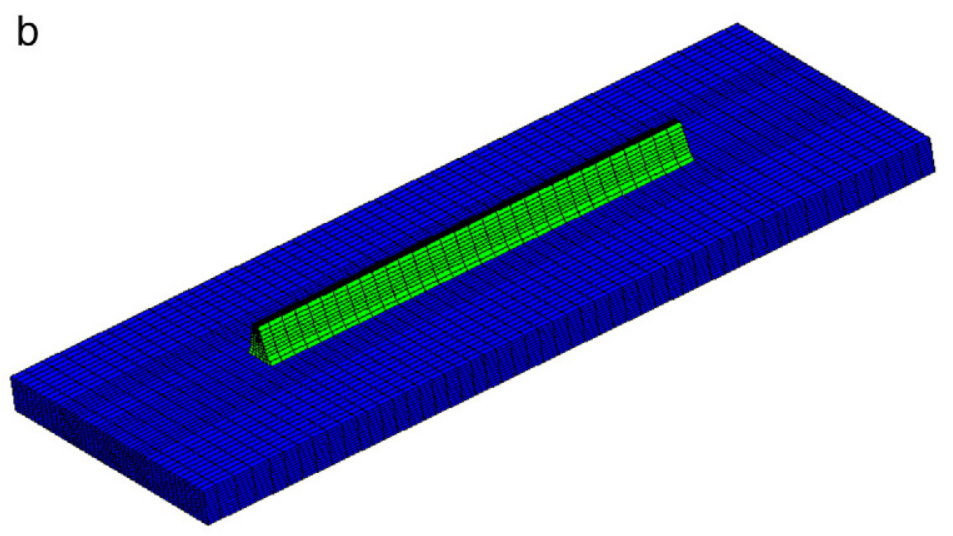

stress indicator has been chosen to check the full stress tensor behavior. Fig. 27 shows a $10 \%$ as average error between the experimental and the numerical results.

An overall good accuracy of the numerical model proposed can be observed.

\section{Conclusions}

This work presents the strategy adopted for the numerical simulation of the SMD processes. A coupled thermo-mechanical framework for the solution of both the energy and momentum balance equations is presented. The appropriate definition of the energy input is discussed when a filler material is used in the welding process. A smooth transition model able to describe the material behavior when the material transforms from liquid (purely viscous) to solid (elasto-plastic) has been presented as an original contribution of this work. Later on, a damage induced porosity model has been introduced to put a limitation to the tensile volumetric strength of the material leading to a hot cracking risk index depending on the phase change shrinkage during the solidification process. The activation procedure to simulate the SMD process along the welding path is also discussed and validated. Later, a number of SMD tests-cases have been carried out at ITP industry. It has been possible to register both the temperature evolution during the full process and the residual stresses after cooling. Distortions have been also measured to be compared with the results achieved by the numerical model. The a

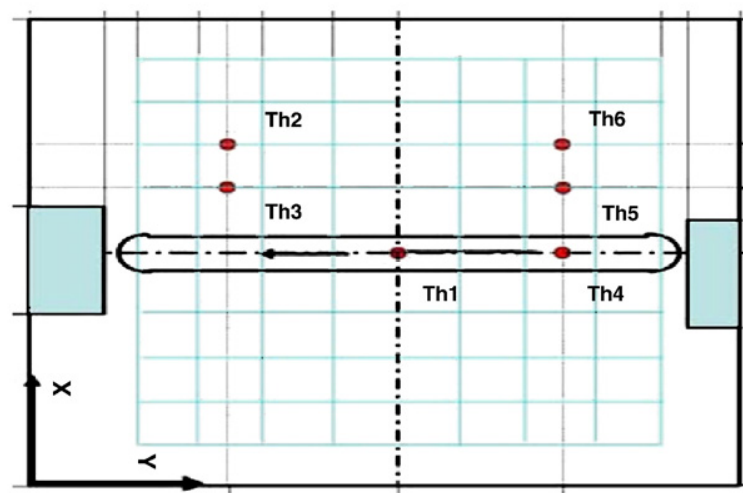

C

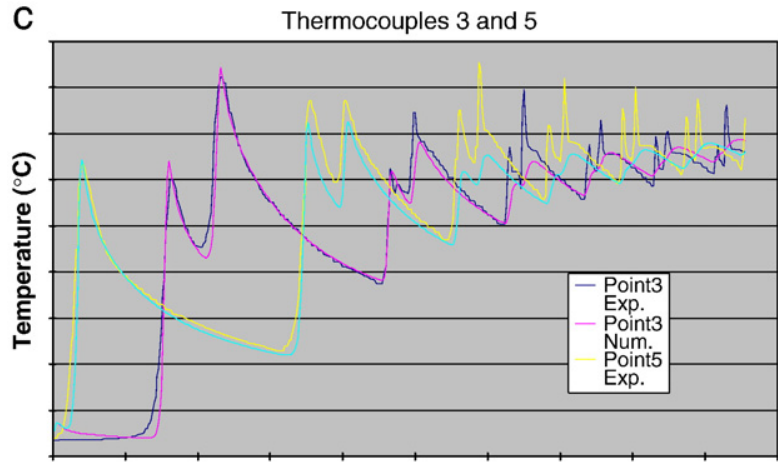

Time (s) b

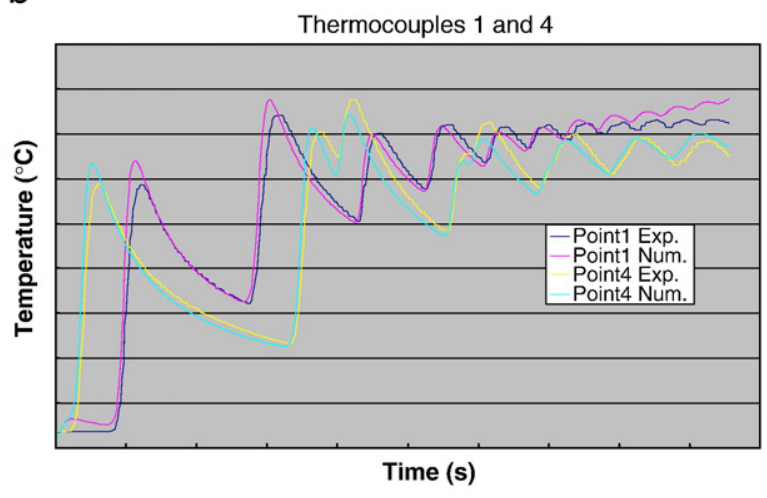

d

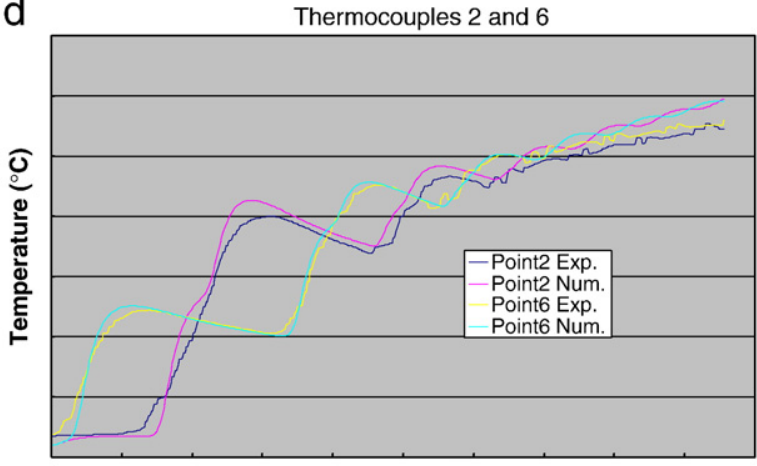

Time (s)

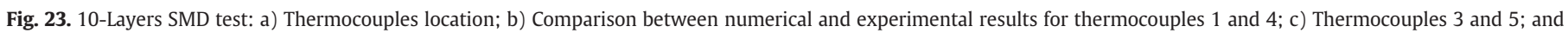
d) Thermocouples 2 and 6 . 


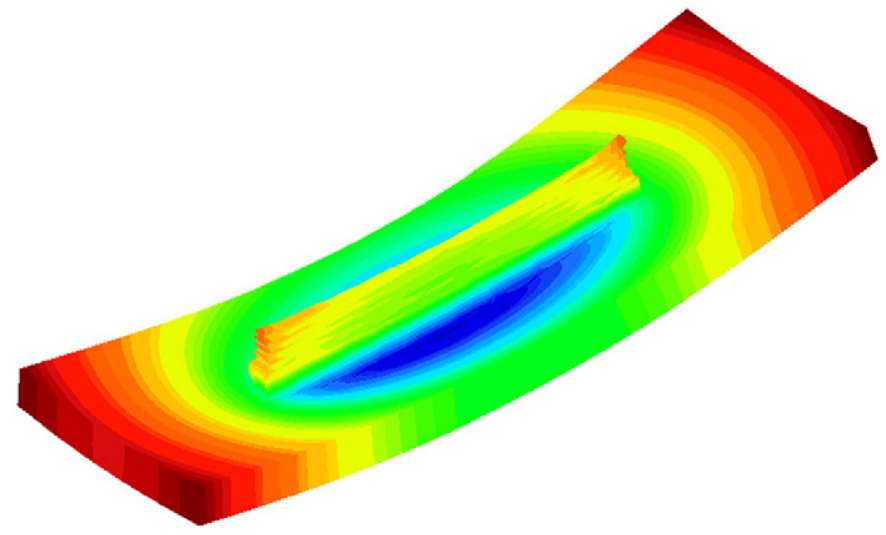

Fig. 24. 10-Layers SMD test: Contour fills of Z-displacement.

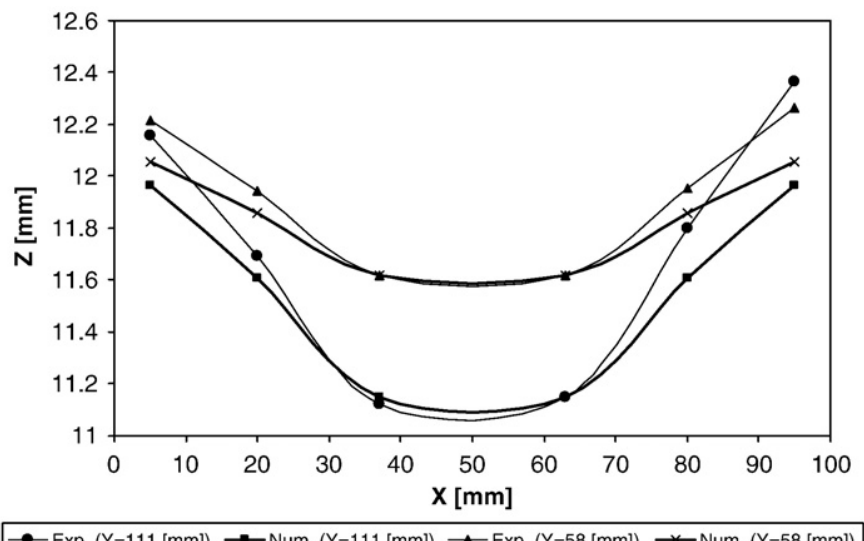

Fig. 25. 10-Layers SMD test: Comparison between numerical and experimental deformation of the transversal section.

benchmarks have shown the accuracy of both the thermal and the mechanical responses when a SMD process must be simulated.

\section{Acknowledgments}

The simulation work carried out by A. Farini is gratefully acknowledged.

Financial support to this work was provided by the European Commission through the Strep-Project 516046: Virtual Engineering for Robust Manufacturing with Design Integration (VERDI), is gratefully acknowledged.

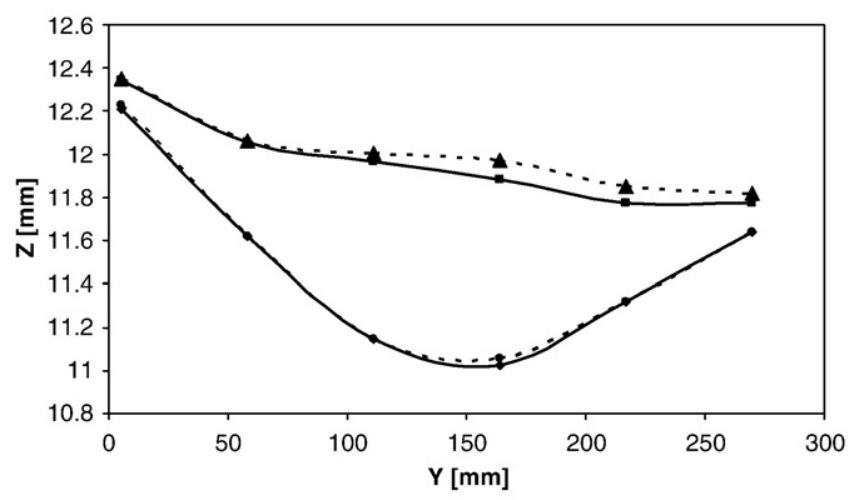

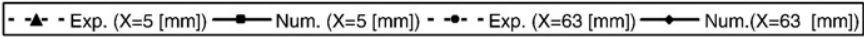

Fig. 26. 10-Layers SMD test: Comparison between numerical and experimental deformation of the longitudinal section.

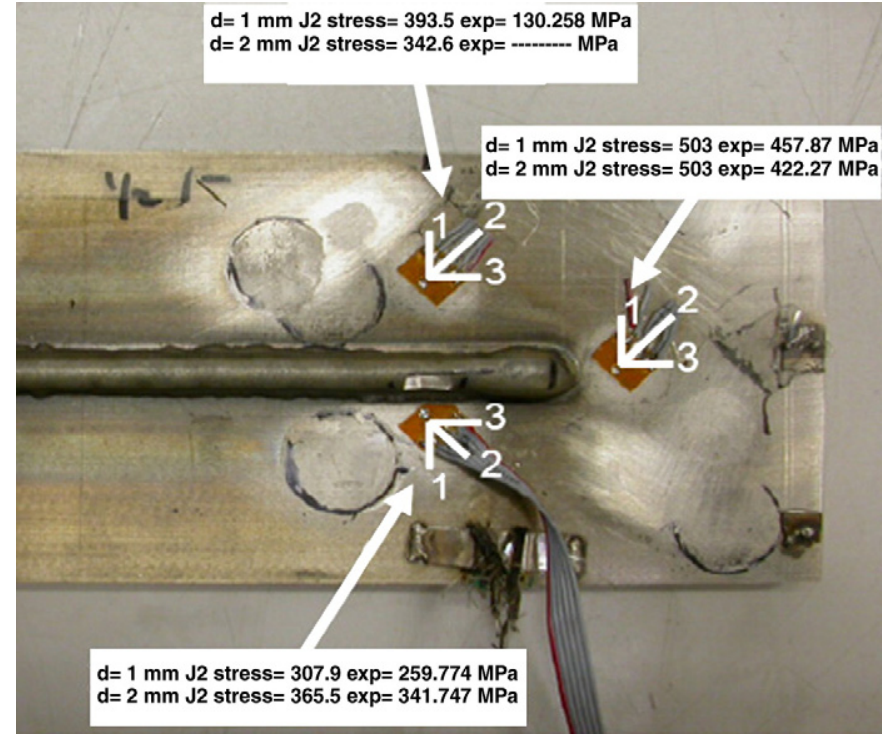

Fig. 27. 10-Layers SMD test: Comparison between numerical and experimental Stresses (von Mises stress) at 3 different strain-gauge locations.

\section{References}

[1] L.-E. Lindgren, H. Runnemalm, M. Näsström, Simulation of multipass welding of a thick plate, Int. J. Numer. Meth. Eng. 44 (1999) 1301-1316.

[2] D.W. Lobitz, J.D. Mc Clure, R.E. Nickell, Residual stresses and distorsions in multi pass welding, Proc. ASME W AM, Numerical Modelling of Manufacturing Processes, PVP-PB-25, 1977, pp. 1-18.

[3] L.-E. Lindgren, Finite element modelling of welding part 1: increased complexity, J. Therm. Stress. 24 (2001) 141-192.

[4] L.-E. Lindgren, Finite element modelling of welding part 2: improved materia modelling, J. Therm. Stress. 24 (2001) 195-231.

[5] L.-E. Lindgren, Finite element modelling of welding part 3: efficiency and integration, J. Therm. Stress. 24 (2001) 305-334.

[6] C. Agelet de Saracibar, M. Cervera, M. Chiumenti, On the formulation of coupled thermoplastic problems with phase-change, Int. J. of Plasticity 15 (1999) $1-34$

[7] M. Cervera, C. Agelet de Saracibar, M. Chiumenti, Thermo-mechanical analysis of industrial solidification processes, Int. J. Numer. Meth. Eng. 46 (1999) 1575-1591.

[8] M. Chiumenti, C. Agelet de Saracibar, M. Cervera, On the numerical modelling of the thermo-mechanical contact for metal casting analysis, J. Heat Transf. 130 (2008) 1-10.

[9] D. Rosenthal, Mathematical theory of heat distribution during welding and cutting, Welding J. 20 (5) (1941) 220-234.

[10] V. Pavelec, R. Tanbakuchi, O.A. Uyehara, P.S. Myers, Welding J. Res. 48 (1969) 295-305 Suppl.

[11] J. Goldak, A. Chakravarti, M. Bibby, A new finite element model for welding heat sources, Metall. Trans. 15B (1984) 299-305.

[12] L.-E. Lindgren, Computational Welding Mechanics: Thermo-mechanical and Microstructural Simulations, August 02, 2007, CRC press.

[13] M. Sundaraman, P. Mukhopadhyay, Carbide precipitation in INCONEL-718, High Temp. Mater. Process II (1-4) (1993) 351-368.

[14] G.A. Knorovsky, M.J. Cieslak, T.J. Headley, A.D. Romig Jr., W.F. Hammetter, INCONEL-718: a solidification diagram, Metall. Trans. A 20 A (1989) 2149-2158.

[15] J.C Simo, T.J.R. Hughes, Computational Inelasticity, Interdisciplinary Applied mathematics, 7, Springer-New York, 1997.

[16] M. Chiumenti, Q. Valverde, C. Agelet de Saracibar, M. Cervera, A stabilized formulation for elasticity using linear displacement and pressure interpolations, Comput. Meth. Appl. Mech. Eng. 191 (2002) 5253-5264.

[17] M. Chiumenti, O Valverde, C. Agelet de Saracibar, M. Cervera, A stabilized formulation for incompressible plasticity using linear triangles and tetrahedra, Int. J. of Plasticity 20 (2004) 1487-1504.

[18] M. Cervera, M. Chiumenti, Q. Valverde, C. Agelet de Saracibar, Mixed linear/linear simplicial elements for incompressible elasticity and plasticity, Comput. Meth. Appl. Mech. Eng. 192 (2003) 5249-5263.

[19] C. Agelet de Saracibar, M. Chiumenti, Q. Valverde, M. Cervera, On the orthogonal subgrid scale pressure stabilization of finite deformation $J 2$ plasticity, Comput. Meth. Appl. Mech. Eng. 195 (2006) 1224-1251.

[20] R. Codina, Stabilization of incompressibility and convection through orthogonal sub-scales in finite element methods, Comput. Meth. Appl. Mech. Eng. 190 (2000) 1579-1599.

[21] R. Codina, Stabilized finite element approximation of transient incompressible flows using orthogonal subscales, Comput. Meth. Appl. Mech. Eng. 191 (2002) 4295-4321. 
[22] F. Brezzi, M. Fortin, Mixed and Hybrid Finite Element Methods, Spinger, New York, 1991.

[23] B. Radhakrishnan, R.G. Thompson, A phase diagram approach to study liquation cracking in alloy 718, Metall. Trans. A 22A (1991) 887-902.

[24] O. Hunzinker, D. Dye, S.M. Roberts, R.C. Reed, A coupled approach for the prediction of solidification cracking during the welding of superalloys, Proc. Conf. on Numerical Analysis of Weldability, Graz-Seggau, Austria, 1999.

[25] D. Deng, H. Murakawa, Numerical simulation of temperature field and residua stress in multi-pass welds in stainless steel pipe and comparison with experimental measurements, Comput. Mater. Sci. 37 (2006) 269-277.

[26] D. Deng, H. Murakawa, W. Liang, Numerical simulation of welding distortion in large structures, Comput. Meth. Appl. Mech. Eng. 196 (2007) 4613-4627.
[27] M.G. Pokorny, C.A. Monroe, C. Beckermann, L. Bichler, C. Ravindran, Prediction of hot tear formation in a magnesium alloy permanent mold casting, Int. J. Metalcasting 2 (2008) 41-53.

[28] J. Lemaitre, J.L. Chaboche, Aspects phénoménologiques de la rupturepar endommagement, J. Méc. Appl. 2 (1978) 317-365 (in French).

[29] J.C. Simó, J.W. Ju, Strain- and stress-based continuum damage models - I: formulation, Int. J. Solids Struct. 23 (1987) 821-840.

[30] D. Clarka, M.R. Bacheb, M.T. Whittaker, Shaped metal deposition of a nickel alloy for aero engine applications, J. Mater. Process. Technol. 203 (1-3) (2008) 439-448.

[31] B. Baufelda, O. Van der Biesta, R. Gault, Additive manufacturing of Ti-6Al-4V components by shaped metal deposition: Microstructure and mechanical properties, Materials and Design. Article in press, Accepted 16 November 2009. 